\title{
Occurrence Et Evaluation Du Risque De L'histamine Dans Les Produits De La Peche Commercialises Sur Le Marche Marocain
}

\author{
Oleya El Hariri (Docteur Vétérinaire) \\ Laboratoire de Biochimie, Biotechnologie, Santé et Environnement, \\ Département de Biologie, Faculté de Science, \\ Université Ibn Tofail Kenitra, Maroc
}

Noureddine Bouchriti (DVM, PhD)

Département de Pathologie et Santé Publique Vétérinaires - Unité HIDAOA, IAV Hassan II, Rabat, Maroc.

\section{Rachid Bengueddour (PhD)}

Laboratoire de Biochimie, Biotechnologie, Santé et Environnement,

Département de Biologie, Faculté de Science,

Université Ibn Tofail Kenitra, Maroc.

doi: 10.19044/esj.2017.v13n27p225 URL:http://dx.doi.org/10.19044/esj.2017.v13n27p225

\section{Abstract}

The histamine levels of preserved and semi-preserved fish marketed in Morocco from 2013 to 2015 have been determined. This study presents the results of histamine control plan of 446 samples of fishery products collected from different areas of Morocco: retail markets, supermarkets, commercial and social restaurants. The synthesis of the data revealed that the occurrence of histamine is low with a non-compliance rate of $3.14 \%$. This rate is $2.02 \%$ for canned sardines and $1.12 \%$ for semi-preserved anchovies. The average histamine values of all batches analyzed did not exceed $5.63 \mathrm{mg} / 100 \mathrm{~g}$. Average values were $5.14 \mathrm{mg} / 100 \mathrm{~g}$ for canned sardine and $7.76 \mathrm{mg} / 100 \mathrm{~g}$ for semi-preserved anchovies. These levels are much lower than the regulatory safety limit. Two qualitative risk assessments of histamine were carried out, one according to the marketing region and the other with reference to the places of marketing. The results showed that the area with the lowest risk is the northern coastal zone with an average of 2.11 $\mathrm{mg} / 100 \mathrm{~g}$ and a non-compliance rate of $0.22 \%$. Low-risk marketing places are the social type restaurants with an average of $4.14 \mathrm{mg} / 100 \mathrm{~g}$ and a noncompliance rate of $0.22 \%$. A ranking of various cities in Morocco was carried out according to histamine content of the marketed fishery products. The results demonstrate the relevance of the official control plan established by the competent Moroccan authority and the control measures implemented 
by the professionals. It can therefore be concluded that preserved and semipreserved fish marketed in Morocco are safe as related to the risk of histamine.

Keywords: Histamine, fish products, canned sardine, semi-preserved anchovies, control plan, risk assessment, Morocco

\section{Résumé}

Les teneurs en histamine des conserves et semi-conserve de poissons commercialisés sur le marché marocain depuis 2013 à 2015 ont été déterminées. Cette étude présente les résultats du plan de contrôle de 1'histamine de 446 échantillons de produits de la pêche prélevés de diverses zones du Maroc, au niveau des points de vente, des grandes surfaces, des lieux de la restauration commerciale et sociale. La synthèse des données a fait ressortir que l'occurrence de l'histamine est faible avec un pourcentage de non-conformité de 3,14\%. Ce taux est de 2,02\% pour les conserves de sardines et $1,12 \%$ pour les semi-conserves d'anchois. La teneur moyenne d'histamine de tous les lots analysés n'a pas dépassé $5,63 \mathrm{mg} / 100 \mathrm{~g}$, elle est de $5,14 \mathrm{mg} / 100 \mathrm{~g}$ pour les conserves de poisson et de $7,76 \mathrm{mg} / 100 \mathrm{~g}$ pour les semi-conserves de poisson, des teneurs largement inférieure à la limite de sécurité préconisée par la réglementation en vigueur.

Deux évaluations qualitatives du risque de l'histamine ont été réalisées, l'une en fonction de la zone de commercialisation et l'autre en fonction des lieux de vente. Les résultats ont montré que la zone présentant le risque le plus faible est la zone côtière nord avec une teneur moyenne de $2,11 \mathrm{mg} / 100 \mathrm{~g}$ et un taux de con conformité de $0,22 \%$. Les lieux de commercialisation à faible risque sont les lieux de la restauration à caractère social avec une teneur moyenne de $4,14 \mathrm{mg} / 100 \mathrm{~g}$ et un taux de non-conformité de $0,22 \%$. Un classement de diverses villes du Maroc a été effectué selon les teneurs d'histamine des produits de la pêche qui y sont commercialisés. Ces résultats témoignent de la pertinence du contrôle officiel diligenté par l'autorité compétente marocaine et des autocontrôles mis en place par les professionnels. On peut avancer donc, que le consommateur marocain est pour le moment protégé contre le risque histamine.

Mots-clés : Histamine, produits de la pêche, conserve de sardine, semi conserve d'anchois, plans de contrôle, évaluation du risque, Maroc

\section{Introduction}

Le Maroc dispose d'un littoral qui s'étend sur plus de $3500 \mathrm{~km}$ de côte dans l'océan Atlantique et la mer Méditerranée, un espace maritime d'environ 1,2 million de $\mathrm{km}^{2}$. Le potentiel halieutique est estimé par la FAO 
à près de 1,5 million de tonnes renouvelables tous les ans. La pêche maritime génère une production annuelle de plus d'un million de tonnes et place le Maroc au premier rang des producteurs africains et au $25^{\text {ème }}$ à l'échelle mondiale (Ouadghiri, 2016).

Le secteur de la pêche présente une ressource structurante de l'économie du Maroc et constitue l'un des piliers de l'économie nationale avec une contribution au PIB évaluée à $2,3 \%$ en moyenne durant les 10 dernières années. En 2015, le secteur halieutique a enregistré un chiffre d'affaire à l'export d'environ 1,85 milliards \$US. Il assure 6,8\% des exportations totales et $58 \%$ des exportations de produits agroalimentaires (Trade, 2016).

Le secteur s'est doté d'une stratégie intégrée, ambitieuse et globale de développement à l'horizon 2020 baptisée «Halieutis». Elle vise la mise à niveau et la modernisation des différents segments du secteur de la pêche ainsi que l'amélioration de sa compétitivité et de sa performance.

Par ailleurs, et en vue d'assurer une meilleure protection de la santé du consommateur, un nouveau dispositif de contrôle a été créé au Maroc en 2010: l'Office National de Sécurité Sanitaire des produits Alimentaires (ONSSA). Cet Office exerce pour le compte de l'État les attributions relatives à la protection de la santé du consommateur et à la préservation de la santé des animaux et des végétaux. Il a pour objectif d'assurer un niveau de sécurité sanitaire élevé tout au long de la chaîne alimentaire en se basant sur une analyse permanente des risques que pourraient représenter les matières premières, les méthodes d'exploitation, et les activités de traitement des denrées alimentaires ainsi que les risques liés aux maladies animales et végétales (ONSSA, 2016).

Parmi les mesures de maitrise et de gestion des risques sanitaires adoptés par cet office figurent la mise en œuvre des plans de contrôle et de surveillance (PS-PC), en vue de prévenir tout dommage sur la santé ou sur la vie des humains ou des animaux. Un plan de surveillance a pour objectif principal l'évaluation globale de l'exposition du consommateur à un risque. Il est toujours fondé sur un échantillonnage réalisé de manière aléatoire au sein d'une population ou d'une sous-population identifiée. Un plan de contrôle a pour objectif principal la recherche d'anomalies, de nonconformités, voire des fraudes. Il est normalement fondé sur un échantillonnage ciblé ou suspect, c'est-à-dire que les prélèvements sont réalisés sur la base de critères de ciblage prédéterminés. Ces plans constituent à la fois une vérification de la rigueur des contrôles réalisés par l'autorité compétente, et l'efficacité des plans de maîtrise sanitaire mis en place par les exploitants du secteur alimentaire.

Annuellement, l'ONSSA met en œuvre un ensemble de PS-PC pour évaluer l'exposition du consommateur aux risques ainsi que la qualité de la 
production nationale. Parmi les plans de contrôle adoptés figure celui de 1'histamine qui a démarré en 2012, et dont les prélèvements concernent les produits de la pêche commercialisés au niveau national. En vue d'harmoniser leur mise en œuvre par les Services Vétérinaires des Directions Régionales, ces plans sont expliqués par des procédures établies et coordonnées par la Direction Centrale de l'ONSSA, détaillant le plan d'échantillonnage, le calendrier et les modalités de réalisation, d'envoi des prélèvements aux laboratoires, les méthodes d'analyse, l'interprétation des résultats, l'échéancier de transmission des résultats et enfin les mesures à prendre en cas de détection de non-conformités.

L'histamine est une amine biogène produite après la mort du poisson sous l'action de certaines bactéries dotées d'enzymes décarboxylases qui transforment l'histidine libre en histamine. L'histidine est un acide aminé présent à des teneurs élevées dans la chair de certaines espèces de poisson.

Pour la production et l'accumulation de l'histamine dans la chair du poisson en post-mortem, trois conditions sont nécessaires (Olley \& Lehane, 2000):

une teneur suffisamment élevée en histidine libre dans la chair du poisson ;

- la présence de bactéries capables de produire l'enzyme histidine décarboxylase ;

- des conditions environnementales favorables (telles des températures élevées) permettant à de telles bactéries de proliférer.

L'histamine est responsable d'intoxication histaminique dont la période d'incubation est courte : de quelques minutes jusqu'à 2 heures. Les symptômes d'empoisonnement à l'histamine (FAO/WHO, 2013) varient entre les individus ; les principaux sont d'ordre :

Cutané (prurit, urticaire, œdème, et inflammation localisée de la peau, éruption cutanée) ;

- $\quad$ Digestif (nausées, vomissements, crampes abdominales et diarrhée) ;

- Hémodynamique (hypotension) ;

- Neurologiques (maux de tête soudains, picotements, sensation de goût poivré, picotement de la bouche et des lèvres et des bouffées de chaleur et transpiration, démangeaisons, difficulté respiratoire sévère). Le signe le plus constant est une rougeur du visage et du cou causant la chaleur et l'inconfort, qui peut ressembler à un coup de soleil.

Des complications plus graves telles que des palpitations cardiaques se produisent, mais sont rares (Taylor, 1986; Olley \& Lehane, 2000). Les symptômes sont considérés comme rarement, sinon jamais d'issue fatale (FAO/WHO, 2013).

La dose toxique pour l'histamine dans le poisson n'est pas encore connue avec précision (Taylor, 1986). Dans la plupart des cas, les niveaux 
d'histamine causant des intoxications suite à la consommation de poisson, ont été au-dessus de $200 \mathrm{mg} / \mathrm{kg}$, souvent supérieures à $500 \mathrm{mg} / \mathrm{kg}$ (FDA, 2011).

Le Règlement (CE) n²073/2005 de la Commission du 15 novembre 2005 concernant les critères microbiologiques applicables aux denrées alimentaires, mentionne spécifiquement les familles et les espèces de poissons associées à des quantités élevées d'histidine. Ce sont les scombridés, les clupéidés, les Engraulidés, les Coryphaenidés, les Pomatomidés et les Scombresocidés (CE, 2005).

L'objectif de ce travail est de faire une synthèse des résultats des plans de contrôle de l'histamine pour une durée de 3 ans (2013, 2014 et 2015), et ce pour évaluer l'occurrence de cette amine biogène dans les produits de pêche commercialisés au niveau national et de statuer ainsi sur le niveau d'efficacité des autocontrôles mis en place par les établissements de traitement des produits de la pêche. Les principaux aspects traités dans cette étude sont :

- Les teneurs moyennes en histamine et les niveaux de nonconformités dans les conserves de poisson et les semi-conserves de poisson commercialisés au Maroc ;

- L'évaluation qualitative du « risque histamine » pour les diverses zones du Maroc (zones côtières, et zone continentale);

- L'évaluation qualitative du « risque histamine » pour les divers lieux de commercialisation (points de vente, grandes surfaces, restauration commerciale, et restauration sociale) ;

- $\quad$ Le classement des villes du Maroc en fonction des teneurs moyennes d'histamine dans les conserves et les semi-conserves de poisson.

\section{Matériels et méthodes}

\section{Échantillonnage}

Cette étude concerne 446 prélèvements dont 362 (81\%) de conserves et $84(19 \%)$ de semi-conserves de poissons (Tableau 1). Un prélèvement est constitué de 9 échantillons. Ces échantillons ont été prélevés auprès de divers points de vente, grandes surfaces, lieux de restauration commerciale et de restauration sociale à travers le Maroc. Ils sont stockés et acheminés à une température ambiante pour les conserves de poisson, alors que les échantillons de semi conserve ont été stockés et transportés dans le respect de la température réglementaire $\left(<+15^{\circ} \mathrm{C}\right)$. Ces prélèvements ont été analysés au niveau des laboratoires officiels de l'ONSSA. 
Tableau 1 : Répartition des prélèvements par type de produit et par année

\begin{tabular}{|c|c|c|}
\hline \multirow{2}{*}{ Année } & Types d'échantillons & $\begin{array}{c}\text { Nombre d'échantillons analysés } \\
\text { dans le cadre du plan de contrôle }\end{array}$ \\
\hline \multirow{3}{*}{2013} & Conserves & 112 \\
\cline { 2 - 3 } & Semi-conserves & 25 \\
\cline { 2 - 3 } & Total & 137 \\
\hline \multirow{3}{*}{2014} & Conserves & 135 \\
\cline { 2 - 3 } & Semi-conserves & 28 \\
\cline { 2 - 3 } & Total & 163 \\
\hline \multirow{3}{*}{2015} & Conserves & 115 \\
\cline { 2 - 3 } & Semi-conserves & 31 \\
\cline { 2 - 3 } & Total & 146 \\
\hline \multicolumn{2}{|c}{} & 446 \\
\hline
\end{tabular}

\section{Analyses de l'histamine}

$\mathrm{Au}$ niveau des laboratoires officiels, seules les méthodes officielles référencées sont utilisées pour réaliser ces analyses. C'est ainsi que la méthode Spectrofluorométrique de Lerck et Bell (1978) ou la méthode de chromatographie liquide à haute performance (HPLC) sont utilisées pour déterminer les teneurs d'histamine. Les résultats ont été collectés auprès de divers services vétérinaires et de divers laboratoires officiels de l'ONSSA.

\section{Résultats Et Discussions}

\section{Synthèse des résultats des plans de contrôle}

La synthèse a concerné 137 résultats d'analyse de produits de la pêche prélevés en 2013, 163 en 2014 et 146 en 2015, au niveau de divers points de vente, grandes surfaces, restauration commerciale et restauration sociale à travers le Maroc. Au total, 362 échantillons de conserves de poisson et 84 échantillons de semi conserves d'anchois ont été synthétisés pour la teneur en histamine.

Pour juger qu'un lot est acceptable en matière d'histamine, sur les neuf échantillons prélevés indépendamment de chaque lot, il faut que :

- $\quad$ La concentration moyenne en histamine soit inférieure à $100 \mathrm{mg} / \mathrm{kg}$;

- Au maximum deux échantillons sur la base des 9 peuvent avoir des teneurs en histamine comprises entre 100 et $200 \mathrm{mg} / \mathrm{kg}$;

- $\quad$ Aucun échantillon ne doit avoir une teneur en histamine supérieure à $200 \mathrm{mg} / \mathrm{kg}$.

Cette synthèse a fait ressortir qu'au fil des années, la moyenne des teneurs en histamine des produits commercialisés au Maroc a diminué, passant d'une moyenne de 5,76 mg/100g en 2013, à 5,74 mg/100g en 2014 pour atteindre une valeur moyenne de $5,4 \mathrm{mg} / 100 \mathrm{~g}$ en 2015 . De même, toutes ces moyennes sont largement inférieures aux limites réglementaires en vigueur. 
La teneur moyenne en histamine dans les produits commercialisés au Maroc, est comprise entre 1 et 7,5 mg/100g, en deçà de la limite réglementaire en vigueur (Figure 1).

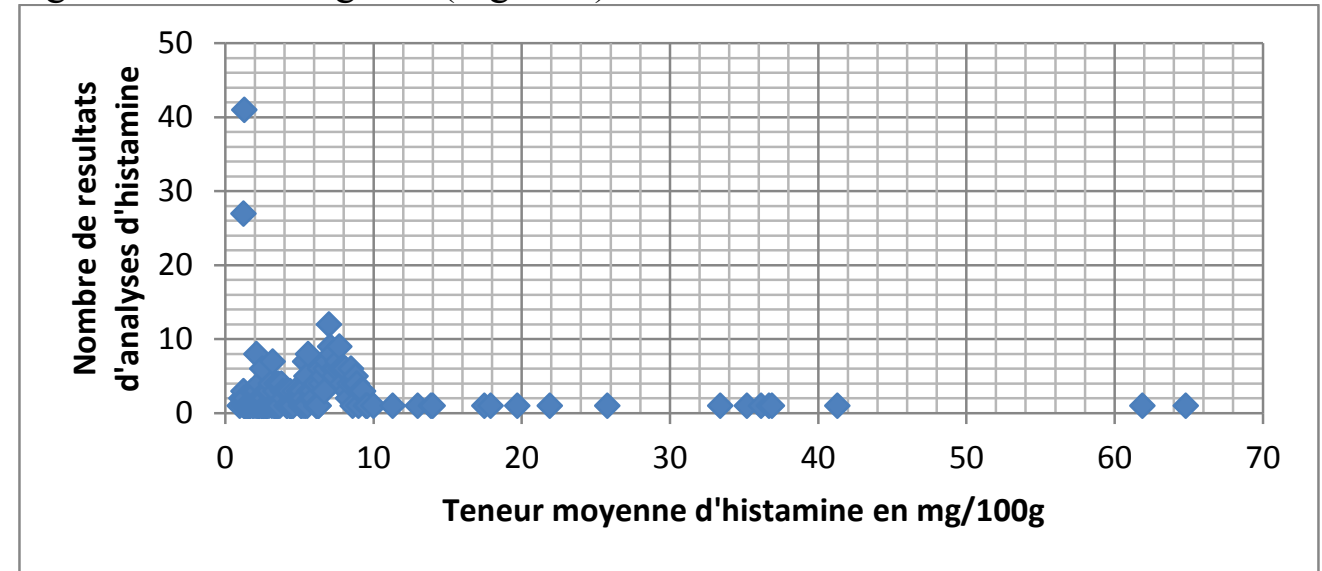

Figure 1 : Répartition des teneurs moyennes de l'histamine

La moyenne des teneurs en histamine des conserves de sardines commercialisées au Maroc est de 5,14 $\mathrm{mg} / 100 \mathrm{~g}$ avec un taux de non conformité (TNC) de $2,02 \%$ sur les 362 échantillons analysés ; celle de la semi-conserve est de 7,76 mg/100g avec un TNC de 1,12\% (Tableau 2).

Tableau 2: Teneurs moyennes en histamine en fonction des types de produits

\begin{tabular}{|c|c|c|c|c|}
\hline Type de Produits & Nombre & Nombre de NC & Taux de NC \% & Moyenne mg/100g \\
\hline Conserve & 362 & 9 & 2,02 & 5,14 \\
\hline Semi-conserve & 84 & 5 & 1,12 & 7,76 \\
\hline Total général & 446 & 14 & 3,14 & 5,63 \\
\hline
\end{tabular}

\section{Répartition des teneurs d'histamine en classes}

$77,58 \%$ des produits commercialisés au Maroc ont une teneur en histamine inférieure à 7,5 mg/100g (Figure 2) et 95,96\% ont des teneurs inferieures à $100 \mathrm{mg} / 100 \mathrm{~g}$. C'est ainsi que $28,7 \%$ ont des teneurs inférieures à $2,5 \mathrm{mg} / 100 \mathrm{~g}, 20,2 \%$ comprises entre 2,5 et $5 \mathrm{mg} / 100 \mathrm{~g}$ et $28,7 \%$ ont des teneurs comprises entre 5 et $7,5 \mathrm{mg} / 100 \mathrm{~g}$. Une très faible proportion $(1,79 \%)$ ont des teneurs entre 10 et $20 \mathrm{mg} / 100 \mathrm{~g}$ et seulement $2,24 \%$ ont des teneurs supérieures au seuil d'acceptabilité qui est de $20 \mathrm{mg} / 100 \mathrm{~g}$ (Tableau3). Ainsi, la presque totalité des produits commercialisés au niveau national présente des teneurs conformes et ne présente pas de risque pour le consommateur marocain. 
Tableau 3: Répartition des teneurs moyennes en histamine par classes

\begin{tabular}{|l|l|l|}
\hline $\begin{array}{l}\text { Classe des teneurs en } \\
\text { histamine }\end{array}$ & $\begin{array}{l}\text { Nombre de Résultats d'histamine } \\
(\mathbf{m g} / \mathbf{1 0 0 g})\end{array}$ & $\begin{array}{l}\text { Pourcentag } \\
\text { e }\end{array}$ \\
\hline $0-2,5 \mathrm{mg} / 100 \mathrm{~g}$ & $\mathbf{1 2 8}$ & $\mathbf{2 8 , 7 0 \%}$ \\
\hline $2,5-5 \mathrm{mg} / 100 \mathrm{~g}$ & $\mathbf{9 0}$ & $\mathbf{2 0 , 1 8 \%}$ \\
\hline $5-7,5 \mathrm{mg} / 100 \mathrm{~g}$ & $\mathbf{1 2 8}$ & $\mathbf{2 8 , 7 0 \%}$ \\
\hline $7,5-10 \mathrm{mg} / 100 \mathrm{~g}$ & $\mathbf{8 2}$ & $\mathbf{1 8 , 3 9 \%}$ \\
\hline $10-20 \mathrm{mg} / 100 \mathrm{~g}$ & $\mathbf{8}$ & $\mathbf{1 , 7 9 \%}$ \\
\hline$>20 \mathrm{mg} / 100 \mathrm{~g}$ & $\mathbf{1 0}$ & $\mathbf{2 , 2 4 \%}$ \\
\hline
\end{tabular}

\section{Répartition des teneurs d'histamine en fonction des zones}

Pour ce faire, nous avons procédé à la répartition des villes du Maroc selon leurs emplacements géographiques en 4 zones: Zone côtière Nord, Zone côtière Centre, Zone continentale et Zone côtière Sud.

La moyenne nationale, tout produit et toute zone confondus, pour les trois années est de l'ordre de 5,63 mg/100g. Elle est de 5,14 mg/100g pour les conserves de poisson et de 7,76 mg/100g pour les semi-conserves.

Ces résultats sont largement inférieurs à ceux trouvés par Ababouch (1989), qui a évalué les taux de contamination des conserves de poisson au Maroc et a trouvé des teneurs allant de 0 à $694 \mathrm{mg} / 100 \mathrm{~g}$, alors que dans la présente étude, les teneurs de l'histamine varient entre 1 et $41,3 \mathrm{mg} / 100 \mathrm{~g}$. Le même auteur a noté une moyenne de $10 \mathrm{mg} / 100 \mathrm{~g}$ et un écart type de 55,28 $\mathrm{g} / 100 \mathrm{~g}$, alors qu'au cours de cette étude la moyenne est de $5,14 \mathrm{mg} / 100 \mathrm{~g}$ et une variance de $21 \mathrm{mg} / 100 \mathrm{~g}$. Cette dichotomie constitue un indice d'une amélioration des conditions d'hygiène et de maîtrise du danger histamine au niveau des filières de transformation des produits de la pêche au niveau national.

Tableau 4 : Répartition des teneurs moyennes d'histamine $(\mathrm{mg} / 100 \mathrm{~g})$ pour les conserves et semi-conserves en fonction des zones

\begin{tabular}{|c|c|c|c|c|c|c|c|c|c|c|c|c|c|c|c|}
\hline \multirow[b]{2}{*}{ Année } & \multicolumn{5}{|c|}{ Conserve } & \multicolumn{5}{|c|}{ Semi-conserve } & \multicolumn{5}{|c|}{ Total } \\
\hline & $\begin{array}{l}\text { M } \\
\text { oy }\end{array}$ & $\begin{array}{l}\mathrm{Nb} \\
\mathrm{re}\end{array}$ & $\begin{array}{c}\mathrm{Ma} \\
\mathrm{X}\end{array}$ & $\begin{array}{c}\mathrm{Mi} \\
\mathrm{n}\end{array}$ & $\begin{array}{l}\mathrm{V} \\
\text { ar }\end{array}$ & $\begin{array}{l}\text { M } \\
\text { oy }\end{array}$ & $\begin{array}{c}\mathrm{Nb} \\
\mathrm{re}\end{array}$ & $\begin{array}{c}\mathrm{Ma} \\
\mathrm{x}\end{array}$ & $\begin{array}{c}\mathrm{Mi} \\
\mathrm{n}\end{array}$ & Var & $\begin{array}{l}\text { M } \\
\text { oy }\end{array}$ & $\begin{array}{c}\mathrm{Nb} \\
\mathrm{re}\end{array}$ & $\begin{array}{c}\mathrm{Ma} \\
\mathrm{x}\end{array}$ & $\begin{array}{c}\mathrm{Mi} \\
\mathrm{n}\end{array}$ & Var \\
\hline 2013 & $\begin{array}{c}5,7 \\
2\end{array}$ & $\begin{array}{c}11 \\
2\end{array}$ & $\begin{array}{c}36 \\
9\end{array}$ & $\begin{array}{c}1,2 \\
3\end{array}$ & 26 & $\begin{array}{c}5,9 \\
5\end{array}$ & 25 & $\begin{array}{c}19, \\
7\end{array}$ & $\begin{array}{l}1, \\
2\end{array}$ & $\begin{array}{c}15 \\
4\end{array}$ & $\begin{array}{c}5,7 \\
6\end{array}$ & $\begin{array}{c}13 \\
7\end{array}$ & $\begin{array}{c}36, \\
9\end{array}$ & $\begin{array}{c}1,2 \\
3\end{array}$ & $\begin{array}{l}23, \\
69\end{array}$ \\
\hline $\begin{array}{c}\text { Zone } \\
\text { Continentale }\end{array}$ & $\begin{array}{c}4,7 \\
9\end{array}$ & 43 & 8,2 & $\begin{array}{c}1,6 \\
8\end{array}$ & 4 & $\begin{array}{c}7,3 \\
8\end{array}$ & 4 & 8,5 & $\begin{array}{c}6, \\
5\end{array}$ & 0,7 & $\begin{array}{c}5,0 \\
1\end{array}$ & 47 & 8,5 & $\begin{array}{c}1,6 \\
8\end{array}$ & $\begin{array}{c}4,2 \\
4\end{array}$ \\
\hline $\begin{array}{l}\text { Zone Côtière } \\
\text { Centre }\end{array}$ & $\begin{array}{c}8,4 \\
7\end{array}$ & 40 & $\begin{array}{c}36, \\
9\end{array}$ & $\begin{array}{c}1,2 \\
3\end{array}$ & 52 & $\begin{array}{c}6,8 \\
2\end{array}$ & 10 & 8,9 & $\begin{array}{l}3, \\
4\end{array}$ & 3,2 & $\begin{array}{c}8,1 \\
4\end{array}$ & 50 & $\begin{array}{c}36, \\
9\end{array}$ & $\begin{array}{c}1,2 \\
3\end{array}$ & $\begin{array}{c}42, \\
7\end{array}$ \\
\hline $\begin{array}{l}\text { Zone Côtière } \\
\text { Nord }\end{array}$ & $\begin{array}{c}1,3 \\
2\end{array}$ & 14 & $\begin{array}{c}2,2 \\
6\end{array}$ & $\begin{array}{c}1,2 \\
3\end{array}$ & $\begin{array}{c}0, \\
1\end{array}$ & $\begin{array}{c}1,3 \\
5\end{array}$ & 5 & 1,8 & $\begin{array}{l}1, \\
2\end{array}$ & 0,1 & $\begin{array}{c}1,3 \\
3\end{array}$ & 19 & $\begin{array}{c}2,2 \\
6\end{array}$ & $\begin{array}{c}1,2 \\
3\end{array}$ & $\begin{array}{c}0,0 \\
7\end{array}$ \\
\hline $\begin{array}{c}\text { Zone Côtière } \\
\text { Sud } \\
\end{array}$ & $\begin{array}{c}5,1 \\
7 \\
\end{array}$ & 15 & 7 & 3,2 & $\begin{array}{l}1, \\
8\end{array}$ & 7,4 & 6 & $\begin{array}{c}19, \\
7 \\
\end{array}$ & $\begin{array}{l}3, \\
4 \\
\end{array}$ & $\begin{array}{c}40 \\
8 \\
\end{array}$ & $\begin{array}{c}5,8 \\
1 \\
\end{array}$ & 21 & $\begin{array}{c}19, \\
7\end{array}$ & 3,2 & $\begin{array}{l}12, \\
56 \\
\end{array}$ \\
\hline 2014 & $\begin{array}{c}4,9 \\
6\end{array}$ & $\begin{array}{c}13 \\
5\end{array}$ & $\begin{array}{c}41, \\
3\end{array}$ & 1,1 & 25 & 9,5 & 28 & $\begin{array}{c}64, \\
8\end{array}$ & $\begin{array}{l}1, \\
2\end{array}$ & $\begin{array}{c}239 \\
, 0\end{array}$ & $\begin{array}{c}5,7 \\
4\end{array}$ & $\begin{array}{c}16 \\
3\end{array}$ & $\begin{array}{c}64, \\
8\end{array}$ & 1,1 & $\begin{array}{l}63, \\
62\end{array}$ \\
\hline $\begin{array}{c}\text { Zone } \\
\text { Continentale }\end{array}$ & $\begin{array}{c}5,9 \\
4\end{array}$ & 46 & $\begin{array}{c}33, \\
4\end{array}$ & 1,7 & 26 & 7 & 7 & 8,9 & $\begin{array}{l}5, \\
5\end{array}$ & 1,5 & $\begin{array}{c}6,0 \\
8\end{array}$ & 53 & $\begin{array}{c}33 \\
4\end{array}$ & 1,7 & $\begin{array}{c}22, \\
9\end{array}$ \\
\hline
\end{tabular}




\begin{tabular}{|c|c|c|c|c|c|c|c|c|c|c|c|c|c|c|c|}
\hline $\begin{array}{c}\text { Zone Côtière } \\
\text { Centre }\end{array}$ & $\begin{array}{c}6,7 \\
6\end{array}$ & 33 & 9,3 & $\begin{array}{c}1,2 \\
3\end{array}$ & $\begin{array}{l}7 \\
4\end{array}$ & $\begin{array}{c}6,7 \\
7\end{array}$ & 9 & 8,9 & $\begin{array}{l}1, \\
3\end{array}$ & 5,2 & $\begin{array}{c}6,7 \\
7\end{array}$ & 42 & 9,3 & $\begin{array}{c}1,2 \\
3\end{array}$ & $\begin{array}{c}6,7 \\
7\end{array}$ \\
\hline $\begin{array}{c}\text { Zone Côtière } \\
\text { Nord } \\
\end{array}$ & $\begin{array}{c}3,6 \\
3 \\
\end{array}$ & 17 & $\begin{array}{c}41, \\
3 \\
\end{array}$ & $\begin{array}{c}1,2 \\
3 \\
\end{array}$ & 94 & $\begin{array}{c}1,2 \\
7 \\
\end{array}$ & 4 & 1,3 & $\begin{array}{l}1, \\
2\end{array}$ & 0,0 & $\begin{array}{c}3,1 \\
8 \\
\end{array}$ & 21 & $\begin{array}{c}41, \\
3 \\
\end{array}$ & $\begin{array}{c}1,2 \\
3 \\
\end{array}$ & $\begin{array}{l}76, \\
29 \\
\end{array}$ \\
\hline $\begin{array}{c}\text { Zone Côtière } \\
\text { Sud }\end{array}$ & $\begin{array}{c}2,8 \\
4\end{array}$ & 39 & 7,9 & 1,1 & $\begin{array}{l}2, \\
6\end{array}$ & $\begin{array}{c}18 \\
9\end{array}$ & 8 & $\begin{array}{c}64, \\
8\end{array}$ & $\begin{array}{l}2, \\
5\end{array}$ & $\begin{array}{c}759 \\
, 9\end{array}$ & $\begin{array}{c}5,5 \\
7\end{array}$ & 47 & $\begin{array}{c}64 \\
8\end{array}$ & 1,1 & $\begin{array}{c}154 \\
, 9\end{array}$ \\
\hline 2015 & 4,8 & $\begin{array}{c}11 \\
5\end{array}$ & $\begin{array}{c}25 \\
8\end{array}$ & 1 & 10 & $\begin{array}{c}7,6 \\
4\end{array}$ & 31 & $\begin{array}{c}36, \\
2\end{array}$ & $\begin{array}{l}1, \\
3\end{array}$ & $\begin{array}{c}75 \\
6\end{array}$ & 5,4 & $\begin{array}{c}14 \\
6\end{array}$ & $\begin{array}{c}36, \\
2\end{array}$ & 1 & $\begin{array}{l}25, \\
15\end{array}$ \\
\hline $\begin{array}{c}\text { Zone } \\
\text { Continentale }\end{array}$ & $\begin{array}{c}4,8 \\
7\end{array}$ & 39 & 8,5 & $\begin{array}{c}1,3 \\
9\end{array}$ & $\begin{array}{l}4, \\
6\end{array}$ & $\begin{array}{c}6,3 \\
2\end{array}$ & 9 & 7,8 & $\begin{array}{l}4, \\
2\end{array}$ & 2,1 & $\begin{array}{c}5,1 \\
5\end{array}$ & 48 & 8,5 & $\begin{array}{c}1,3 \\
9\end{array}$ & $\begin{array}{c}4,3 \\
8\end{array}$ \\
\hline $\begin{array}{c}\text { Zone Côtière } \\
\text { Centre }\end{array}$ & $\begin{array}{c}5,8 \\
9\end{array}$ & 39 & $\begin{array}{c}25, \\
8\end{array}$ & 1,3 & 18 & 8,3 & 9 & $\begin{array}{c}21, \\
9\end{array}$ & $\begin{array}{l}1, \\
3\end{array}$ & $\begin{array}{c}46, \\
6\end{array}$ & $\begin{array}{c}6,3 \\
4 \\
\end{array}$ & 48 & $\begin{array}{c}25 \\
8\end{array}$ & 1,3 & $\begin{array}{l}23, \\
69\end{array}$ \\
\hline $\begin{array}{c}\text { Zone Côtière } \\
\text { Nord }\end{array}$ & $\begin{array}{c}1,8 \\
9\end{array}$ & 16 & 10 & 1 & $\begin{array}{l}4, \\
7\end{array}$ & 1,3 & 5 & 1,3 & $\begin{array}{l}1, \\
3\end{array}$ & 0,0 & $\begin{array}{c}1,7 \\
5\end{array}$ & 21 & 10 & 1 & $\begin{array}{c}3,6 \\
1\end{array}$ \\
\hline $\begin{array}{c}\text { Zone Côtière } \\
\text { Sud }\end{array}$ & $\begin{array}{c}4,8 \\
3\end{array}$ & 21 & 8,5 & $\begin{array}{c}2,2 \\
9\end{array}$ & $\begin{array}{l}2, \\
8\end{array}$ & $\begin{array}{c}12, \\
3\end{array}$ & 8 & $\begin{array}{c}36, \\
2\end{array}$ & $\begin{array}{l}2, \\
6\end{array}$ & $\begin{array}{c}211 \\
, 9\end{array}$ & 6,9 & 29 & $\begin{array}{c}36, \\
2\end{array}$ & $\begin{array}{c}2,2 \\
9\end{array}$ & $\begin{array}{l}66, \\
61\end{array}$ \\
\hline Total général & $\begin{array}{c}5,1 \\
4\end{array}$ & $\begin{array}{c}36 \\
2\end{array}$ & $\begin{array}{c}41, \\
3\end{array}$ & 1 & 21 & $\begin{array}{c}7,7 \\
6\end{array}$ & 84 & $\begin{array}{c}64, \\
8\end{array}$ & $\begin{array}{l}1, \\
2\end{array}$ & $\begin{array}{c}111 \\
, 5\end{array}$ & $\begin{array}{c}5,6 \\
3\end{array}$ & $\begin{array}{c}44 \\
6\end{array}$ & $\begin{array}{c}64, \\
8\end{array}$ & 1 & $\begin{array}{l}38, \\
62\end{array}$ \\
\hline
\end{tabular}

L'évolution des teneurs moyennes d'histamine durant les années 2013 à 2015 en fonction des zones est présentée dans la figure 2 .

En 2013, la teneur la plus élevée pour les conserves de poisson a été constatée dans la zone côtière centre avec une moyenne de $8,47 \mathrm{mg} / 100 \mathrm{~g}$, suivie par la zone côtière sud avec une teneur moyenne de $5,17 \mathrm{mg} / 100 \mathrm{~g}$, ensuite on trouve la zone continentale avec une teneur moyenne de 4,79 $\mathrm{mg} / 100 \mathrm{~g}$, et en dernier lieu la zone côtière nord avec les teneurs les plus faibles en histamine de $1,32 \mathrm{mg} / 100 \mathrm{~g}$.

Concernant l'année 2014, c'est toujours les conserves de poisson de la zone côtière centre qui montrent la teneur la plus élevée avec une moyenne de $6,76 \mathrm{mg} / 100 \mathrm{~g}$, suivie par la zone continentale avec une valeur de $5,94 \mathrm{mg} / 100 \mathrm{~g}$, ensuite la zone côtière nord avec une moyenne de 3,63 $\mathrm{mg} / 100 \mathrm{~g}$, et en dernier lieu, la zone côtière sud avec une moyenne de 2,84 $\mathrm{mg} / 100 \mathrm{~g}$.

Pour l'année 2015, on retrouve au premier rang la zone côtière centre avec une teneur de $5,89 \mathrm{mg} / 100 \mathrm{~g}$, suivie de la zone continentale avec une teneur moyenne de $4,87 \mathrm{mg} / 100 \mathrm{~g}$, la zone côtière sud en troisième lieu avec $4,83 \mathrm{mg} / 100 \mathrm{~g}$, et en dernier lieu la zone côtière nord avec une teneur de 1,89 $\mathrm{mg} / 100 \mathrm{~g}$.

Entre 2013 et 2015, les teneurs les plus élevées en histamine dans les conserves de poisson sont celles de la zone côtière centre alors que les teneurs en histamine les plus faibles correspondent à la zone côtière nord du Maroc. 


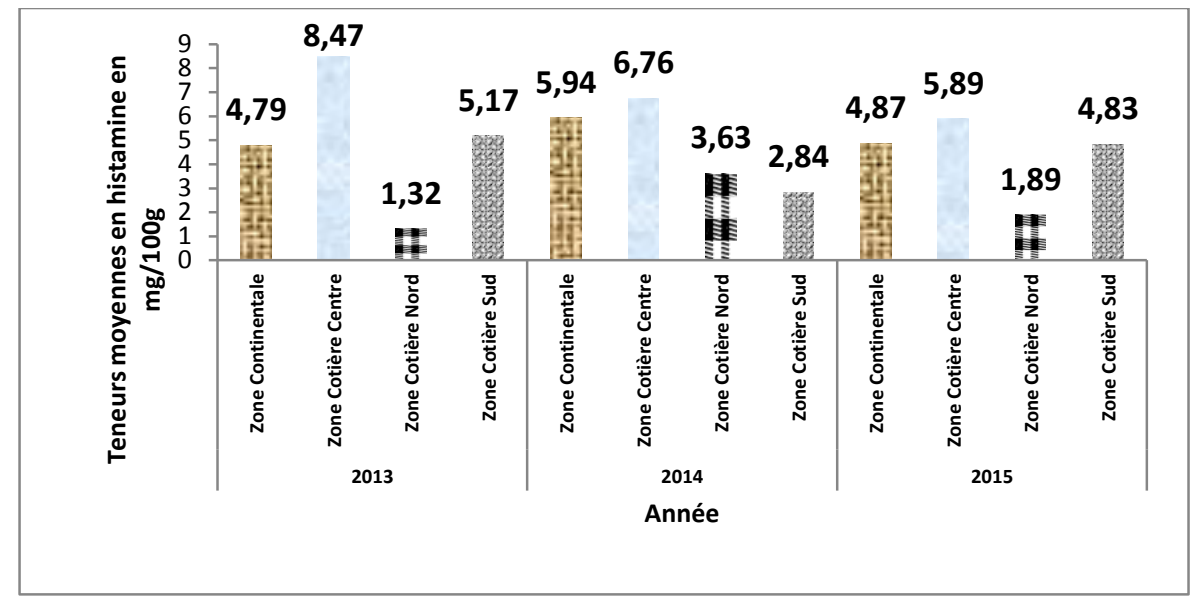

Figure 2 : Teneurs moyennes en histamine dans les conserves en fonction des 4 zones

Pour les semi conserves de poissons, la teneur la plus élevée en histamine est trouvée dans la zone côtière sud avec $18,86 \mathrm{mg} / 100 \mathrm{~g}$ et la moyenne la plus faible est trouvée dans la zone côtière nord avec une valeur de $1,27 \mathrm{mg} / 100 \mathrm{~g}$ (Figure 3).

Durant les trois années, ce sont toujours les semi-conserves de poisson commercialisées dans la zone côtière sud qui ont présenté les teneurs les plus élevées en histamine, alors que les faibles teneurs ont été trouvées dans la zone côtière nord (Figure 3).

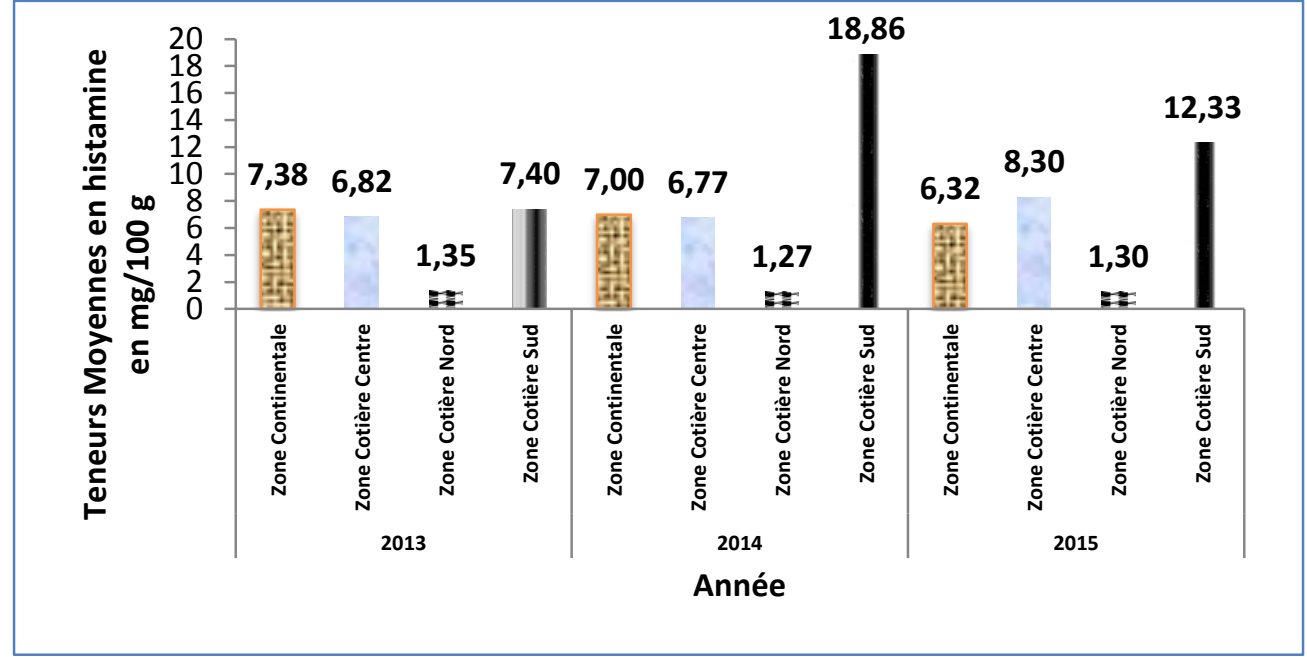

Figure 3 : Teneurs moyennes en histamine des semi-conserves selon les zones.

La répartition des diverses villes du Maroc en zone continentale et en zone côtière est représentée dans le tableau 4 . Elle révèle que les conserves commercialisées dans les zones côtières ont une teneur en histamine plus faibles que celles des zones continentales. 
La teneur moyenne trouvée dans les zones côtières est de $\mathbf{5 , 0 9}$ $\mathbf{m g} / \mathbf{1 0 0 g}$ alors que celle dans la zone continentale est de $\mathbf{5 , 2 3} \mathbf{~ m g} / \mathbf{1 0 0 g}$. Ce qui peut être expliqué par les longues distances de transport que doit parcourir le poisson frais destiné à la transformation qui pourrait résulter en une accumulation plus importante d'histamine dans la matière première.

C'est une tendance inverse retrouvée pour la semi-conserve, où la teneur moyenne en histamine est plus élevée pour la zone côtière $(8,06$ $\mathrm{mg} / 100 \mathrm{~g})$ en comparaison à la zone continentale $(6,77 \mathrm{mg} / 100 \mathrm{~g})$. Ceci pourrait s'expliquer par des pratiques d'entreposage différentes au maillon commercialisation. Les températures des zones continentales sont plus élevées par rapport à celles des zones côtières au Maroc; Vu que les semiconserves de poisson nécessitent une température réglementaire d'entreposage maximale de $+15^{\circ} \mathrm{C}$ et $+4^{\circ} \mathrm{C}$ pour la semi-conserve marinée, on peut supposer que les températures d'entreposage plus élevées sont favorables à une accumulation plus importante d'histamine.

Tableau 5 : Comparaison des teneurs d'histamine entre les zones côtières et la zone continentale

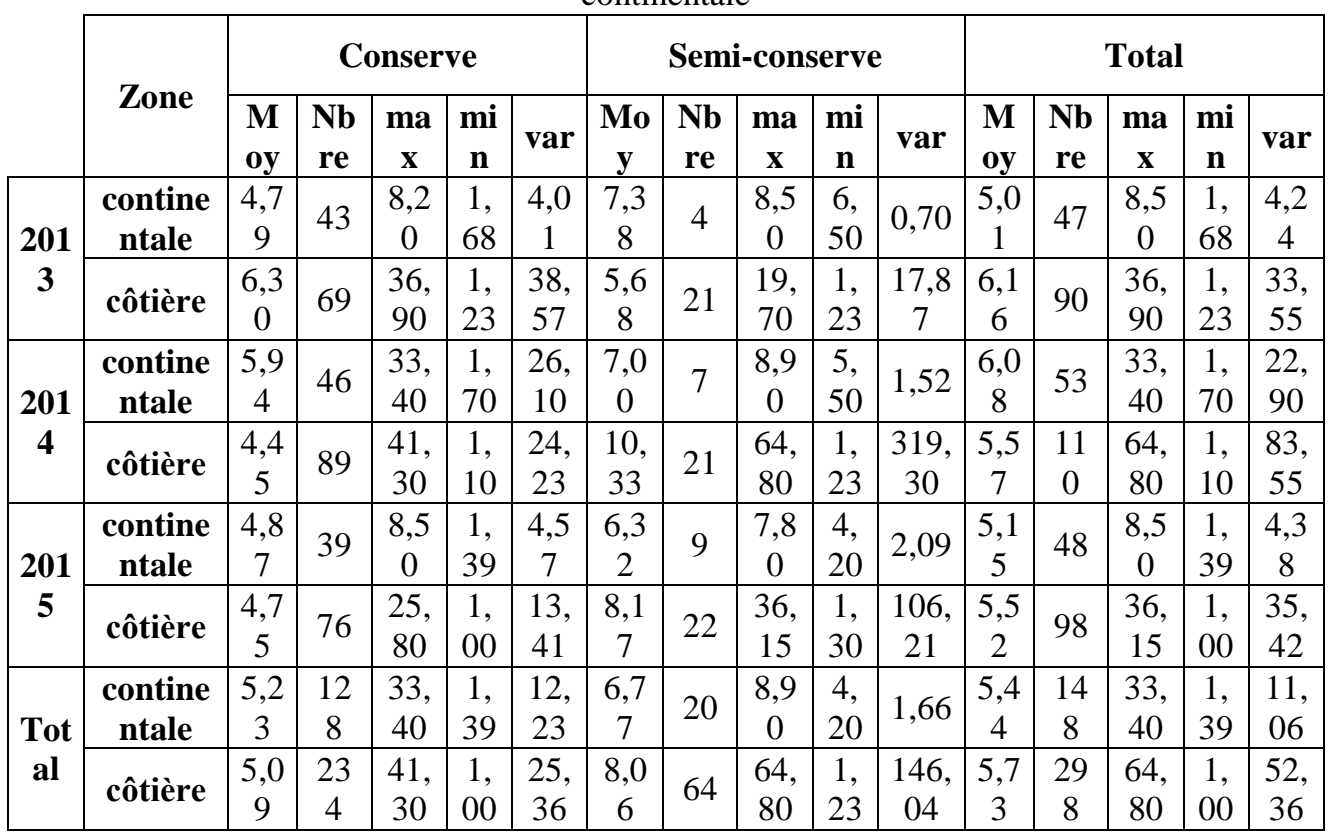

\section{Conformité des échantillons}

Partout dans le monde, des règlements définissent une limite d'histamine à ne pas dépasser dans les poissons. Pour les espèces sensibles associées à une grande quantité d'histidine, la législation marocaine ainsi que celle de l'UE, stipule que pour qu'un un lot soit acceptable, sur les neuf échantillons prélevés indépendamment de chaque lot, il faut que :

la valeur moyenne $(\mathrm{m})$ observée soit inférieure à $100 \mathrm{mg} / \mathrm{kg}$; 
- $\quad$ un maximum de deux échantillons sur la base des 9 peuvent avoir des teneurs comprises entre 100 et $200 \mathrm{mg} / \mathrm{kg}$ (c);

- $\quad$ aucune valeur observée ne dépasse la limite de $200 \mathrm{mg} / \mathrm{kg}(\mathrm{M})$.

Pour les produits de la pêche ayant subi un traitement de Salage/maturation en saumure, et élaborés à partir d'espèces de poissons associées à une grande quantité d'histidine, les valeurs de $\mathrm{m}$ et $\mathrm{M}$ sont portées au double $(\mathrm{m}=200 \mathrm{mg} / \mathrm{kg}$ et $\mathrm{M}=400 \mathrm{mg} / \mathrm{kg})$.

Aux USA, le plan d'échantillonnage de la FDA (2010) est un plan à trois classes de $\mathrm{n}=18, \mathrm{c}=1, \mathrm{~m}=50 \mathrm{mg} / \mathrm{kg}$ et $\mathrm{M}=500 \mathrm{mg} / \mathrm{kg}$.

Les normes du Codex Alimentarius stipulent que les produits des espèces sensibles ne doivent pas contenir plus de $100 \mathrm{mg}$ d'histamine par $\mathrm{kg}$ de chair de poisson qui correspond à la limite de décomposition (FAO/WHO, 2013).

Le pourcentage de non-conformité le plus élevé en matière d'histamine (tous produits confondus) est trouvé dans la zone côtière centre, de l'ordre de $1,57 \%$, suivi par la zone côtière sud où $0,9 \%$ des produits commercialisés sont non conformes, alors que pour la zone continentale, il est de $0,45 \%$ et de $0,22 \%$ pour la zone côtière nord qui vient en dernier lieu en matière de non-conformité durant les trois années (Tableau 6).

Tableau 6: Pourcentage des non-conformités pour l'histamine en fonction des zones

\begin{tabular}{|c|c|c|c|}
\hline Zones & Nombre de Résultats & Somme de NC & Somme de pourcentage NC \% \\
\hline Continentale & 148 & 2 & 0,45 \\
\hline Côtière Centre & 140 & 7 & 1,57 \\
\hline Côtière Nord & 61 & 1 & 0,22 \\
\hline Côtière Sud & 97 & 4 & 0,90 \\
\hline Total général & 446 & 14 & 3,14 \\
\hline
\end{tabular}

\section{Eléments d'évaluation du risque « Histamine » selon les zones du Maroc}

Les teneurs moyennes d'histamine pour chaque zone ont été calculées, le pourcentage des non conformités a été chiffré et le pourcentage des teneurs d'histamine a été ordonnancé en six classes : a $<2,5 \mathrm{mg} / 100 \mathrm{~g}$, $2,5<\mathrm{b}<5 ; \mathrm{mg} / 100 \mathrm{~g}, 5<\mathrm{c}<7,5 \mathrm{mg} / 100 \mathrm{~g}, 7,5<\mathrm{d}<10 \mathrm{mg} / 100 \mathrm{~g}, 10<\mathrm{e}<20$ $\mathrm{mg} / 100 \mathrm{~g}$ et $\mathrm{f}>20 \mathrm{mg} / 100 \mathrm{~g}$ (Tableau 7).

Tableau 7 : Teneurs moyennes en histamine, pourcentage des non conformités ainsi que le pourcentage des teneurs d'histamine par classe pour les 4 zones.

\begin{tabular}{|c|c|c|c|c|c|c|c|c|}
\hline \multirow{2}{*}{ Zones } & \multirow{2}{*}{$\begin{array}{c}\text { Moy } \\
\text { (mg/100g) }\end{array}$} & \multirow{2}{*}{$\begin{array}{l}\mathrm{NC} \\
(\%)\end{array}$} & \multicolumn{6}{|c|}{$\begin{array}{l}\text { Pourcentage des teneurs d'histamine par classe } \\
(\%)\end{array}$} \\
\hline & & & $<2,5$ & ]2,5-5] & $\begin{array}{l}5- \\
7,5]\end{array}$ & $\begin{array}{c}77,5- \\
10]\end{array}$ & $\begin{array}{l}10- \\
20]\end{array}$ & $>20$ \\
\hline Continentale & 5,44 & 0,45 & 4,48 & 10,54 & 11,21 & 6,50 & 0,22 & 0,22 \\
\hline Côtière Centre & 7,11 & 1,57 & 5,61 & 0,90 & 12,11 & 10,76 & 1,12 & 0,90 \\
\hline Côtière Nord & 2,11 & 0,22 & 13,23 & 0,00 & 0,00 & 0,00 & 0,22 & 0,22 \\
\hline Côtière Sud & 6,02 & 0,90 & 5,38 & 8,74 & 5,38 & 1,12 & 0,22 & 0,90 \\
\hline Total & 5,63 & 3,14 & 28,70 & 20,18 & 28,70 & 18,39 & 1,79 & 2,24 \\
\hline
\end{tabular}


Si on considère une graduation allant de 1 à 4 qui correspond aux 4 zones du Maroc, les teneurs moyennes en histamine, les pourcentages des non conformités ainsi que le pourcentage des teneurs d'histamine par classe ont été classés en fonction de ces 4 rangs.

$\mathrm{Si}$ on retient la formule suivante pour le calcul du risque «Histamine » sur une échelle allant de 1 à 100 :

Risque histamine $=$ Rang des teneurs moyennes en histamine $\mathrm{x}$ Rang du taux de nonconformité $\mathrm{x}$ Rang du Pourcentage des teneurs pour toutes les classes

La valeur maximale du risque de l'histamine serait égale à $4^{8}$ et celle minimale serait égale à 1 (Tableau 8 ).

Tableau 8: Evaluation semi-quantitative du risque histamine pour les 4 zones

\begin{tabular}{|c|c|c|c|c|c|c|c|c|c|c|}
\hline \multirow{2}{*}{$\begin{array}{c}\text { Lieu de } \\
\text { prélèvement }\end{array}$} & \multirow{2}{*}{$\begin{array}{c}\text { Rang de } \\
\text { la } \\
\text { Moy }\end{array}$} & \multirow{2}{*}{$\begin{array}{l}\text { Ran } \\
\text { g de } \\
\text { NC } \\
(\%)\end{array}$} & \multicolumn{6}{|c|}{$\begin{array}{l}\text { Rang des pourcentages } \\
\text { des teneurs par chaque } \\
\text { classe }\end{array}$} & \multirow{2}{*}{\begin{tabular}{|c} 
Importanc \\
$\mathrm{e}$ \\
du Risque \\
de \\
l'histamin \\
e
\end{tabular}} & \multirow{2}{*}{$\begin{array}{l}\text { Classe du } \\
\text { risque }\end{array}$} \\
\hline & & & $\begin{array}{l}n \\
\text { vi }\end{array}$ & $\begin{array}{l}n \\
n \\
n \\
n\end{array}$ & $\begin{array}{l}n \\
i \\
i \\
n\end{array}$ & $\begin{array}{l}5 \\
\vdots \\
1 \\
n \\
2 \\
\end{array}$ & 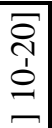 & & & \\
\hline Zone Continentale & 2 & 2 & 1 & 4 & 3 & 3 & 1 & 1 & $2,14 \%$ & $\begin{array}{l}\text { Risque } \\
\text { faible }\end{array}$ \\
\hline $\begin{array}{l}\text { Zone Côtière } \\
\text { Centre }\end{array}$ & 4 & 4 & 3 & 2 & 4 & 4 & 2 & 2 & $91,37 \%$ & $\begin{array}{c}\text { Risque } \\
\text { élevé }\end{array}$ \\
\hline Zone Côtière Nord & 1 & 1 & 4 & 1 & 1 & 1 & 1 & 1 & $0,06 \%$ & $\begin{array}{c}\text { Risque } \\
\text { négligeabl } \\
\mathrm{e}\end{array}$ \\
\hline Zone Côtière Sud & 3 & 3 & 2 & 3 & 2 & 2 & 1 & 2 & $6,42 \%$ & $\begin{array}{l}\text { Risque } \\
\text { moyen }\end{array}$ \\
\hline
\end{tabular}

La méthode utilisée pour l'évaluation semi-quantitative du risque a permis de comparer le niveau de risque par zone.

Les produits de la pêche commercialisés dans la zone côtière centre présentent un risque élevé en comparaison aux autres zones en matière de teneur en histamine, suivis par la zone côtière sud, de la zone continentale, et enfin les produits de la pêche commercialisés dans la zone côtière nord.

\section{Histamine et divers lieux de commercialisation du Maroc}

\section{Teneurs de l'histamine en fonction des lieux de commercialisation}

Les produits de la pêche commercialisés dans les lieux de restauration commerciale ont la moyenne d'histamine la plus élevée $(6,68$ $\mathrm{mg} / 100 \mathrm{~g})$, suivis par ceux vendus dans les points de vente $(6,09 \mathrm{mg} / 100 \mathrm{~g})$, au troisième rang, ceux commercialisés dans les grandes surfaces $(5,77$ $\mathrm{mg} / 100 \mathrm{~g}$ ) et en dernier lieu on trouve les valeurs les plus faibles pour les 
produits de la pêche dans les lieux de restauration collective à caractère sociale (cantines scolaires, hopitaux, ...) (4,14 mg/100g) (Tableau 9).

La moyenne d'histamine la plus élevée a été constatée dans les produits de la pêche commercialisés au niveau des points de vente de la zone côtière centre $(10,3 \mathrm{mg} / 100 \mathrm{~g})$. Ce résultat est en concordance avec celui concernant le risque élevé retrouvé pour cette même zone.

Tableau 9: Teneur d'histamine en fonction des lieux de vente et par zone

\begin{tabular}{|c|c|c|c|c|c|}
\hline Lieux de vente & Nombre & Moyenne & Max & Min & Var \\
\hline Grande surface & 222 & 5,77 & 64,8 & 1 & 46,72 \\
\hline Zone Continentale & 36 & 5,71 & 8,8 & 1,8 & 5,18 \\
\hline Zone Côtière Centre & 110 & 6,38 & 25,8 & 1,23 & 14,84 \\
\hline Zone Côtière Nord & 41 & 1,51 & 10 & 1 & 1,88 \\
\hline Zone Côtière Sud & 35 & 8,89 & 64,8 & 2,1 & 215,45 \\
\hline Point de vente & 116 & 6,09 & 36,9 & 1,1 & 30,85 \\
\hline Zone Continentale & 44 & 7,22 & 33,4 & 2,3 & 18,24 \\
\hline Zone Côtière Centre & 24 & 10,30 & 36,9 & 6 & 71,37 \\
\hline Zone Côtière Nord & 8 & 1,42 & 2,1 & 1,23 & 0,09 \\
\hline Zone Côtière Sud & 40 & 3,27 & 7,9 & 1,1 & 3,76 \\
\hline Restauration Commerciale & 31 & 6,68 & 41,3 & 1,23 & 88,15 \\
\hline Zone Continentale & 1 & 2,70 & 2,7 & 2,7 & --- \\
\hline Zone Côtière Centre & 6 & 7,73 & 8,9 & 6,2 & 1,08 \\
\hline Zone Côtière Nord & 9 & 5,73 & 41,3 & 1,23 & 177,95 \\
\hline Zone Côtière Sud & 15 & 7,09 & 36,15 & 2,1 & 84,45 \\
\hline Restauration Sociale & 77 & 4,14 & 17,9 & 1,3 & 5,74 \\
\hline Zone Continentale & 67 & 4,17 & 17,9 & 1,39 & 6,10 \\
\hline Zone Côtière Nord & 3 & 1,33 & 1,39 & 1,3 & 0,00 \\
\hline Zone Côtière Sud & 7 & 5,09 & 5,8 & 3,8 & 0,58 \\
\hline Total général & 446 & 5,63 & 64,8 & 1 & 38,62 \\
\hline
\end{tabular}

La moyenne la plus faible d'histamine a été observée au niveau des lieux de restauration collective à caractère social $(1,33 \mathrm{mg} / 100 \mathrm{~g})$. Ceci témoigne du fait que les producteurs sont conscients du risque élevé associé à cette catégorie de consommateurs (personnes âgées, malades, enfants en bas âge, personnes à statut immunitaire déficient,...) et prennent le soin de destiner les produits « les plus surs » à ce segment de la restauration. A ceci s'ajoute aussi la stratégie de contrôle renforcé de l'ONSSA pour les produits destinés à ce segment de la restauration et qui trouve ici confirmation à travers nos résultats.

Les produits de la pêche commercialisés dans les grandes surfaces présentent les teneurs en histamine les plus élevées en zone côtière sud $(8,89$ 
$\mathrm{mg} / 100 \mathrm{~g}$ ) et les teneurs les plus faibles en zone côtière nord avec 1,51 $\mathrm{mg} / 100 \mathrm{~g}$. Dans le cas des semi-conserves, ceci pourrait s'expliquer par des fluctuations de température d'entreposage engendrées par les échanges entre les vitrines d'exposition et l'air ambiant au niveau des grandes surfaces. Ce phénomène serait plus accentué au sud en rapport avec des températures plus élevées. Pour les conserves, les différences ne pourraient s'expliquer que par des degrés de maîtrise du danger histamine variables entre les producteurs.

Pour les points de vente, c'est ceux également localisés dans la zone côtière centre qui vendent des produits avec les teneurs les plus élevées en histamine $(10,3 \mathrm{mg} / 100 \mathrm{~g})$ alors que ceux installés dans la zone côtière nord commercialisent les produits ayant les plus faibles teneurs en histamine 1,42 $\mathrm{mg} / 100 \mathrm{~g}$.

Les produits de la pêche commercialisés en restauration commerciale dans la zone côtière centre ont les teneurs les plus élevées en histamine $(7,73$ $\mathrm{mg} / 100 \mathrm{~g}$ ), alors que ceux dans la zone continentale ont les plus faibles teneurs $(2,7 \mathrm{mg} / 100 \mathrm{~g})$.

Concernant les produits de la pêche commercialisés dans les lieux de la restauration sociale, c'est ceux de la zone côtière sud qui ont les teneurs les plus élevées en histamine $(5,09 \mathrm{mg} / 100 \mathrm{~g})$ et ce sont ceux de la zone côtière nord qui ont les plus faibles teneurs $(1,33 \mathrm{mg} / 100 \mathrm{~g})$.

En 2013, la teneur la plus élevée a été trouvée au niveau des produits issus des points de vente, de l'ordre de $9,52 \mathrm{mg} / 100 \mathrm{~g}$, suivi par les grandes surfaces par 5,28 mg/100g, ensuite les lieux de la restauration commerciale avec une moyenne de $5,09 \mathrm{mg} / 100 \mathrm{~g}$ et en dernière position les lieux de la restauration sociale avec une valeur de $4,19 \mathrm{mg} / 100 \mathrm{~g}$ (Figure 4).

En 2014 et 2015, ce sont les échantillons issus des lieux de la restauration commerciale qui ont des teneurs élevées en histamine de l'ordre de 7,41 et 9,02 mg/100g, respectivement, suivis par les grandes surfaces par des teneurs de 6,49 et $5,53 \mathrm{mg} / 100 \mathrm{~g}$, respectivement, ensuite les points de vente par 4,96 et 5,43mg/100g et en dernier lieu, se classent les lieux de la restauration sociale qui ont distribué des produits de la pêche avec les plus faibles teneurs en histamine de l'ordre de 4,59 et $3,35 \mathrm{mg} / 100 \mathrm{~g}$. 


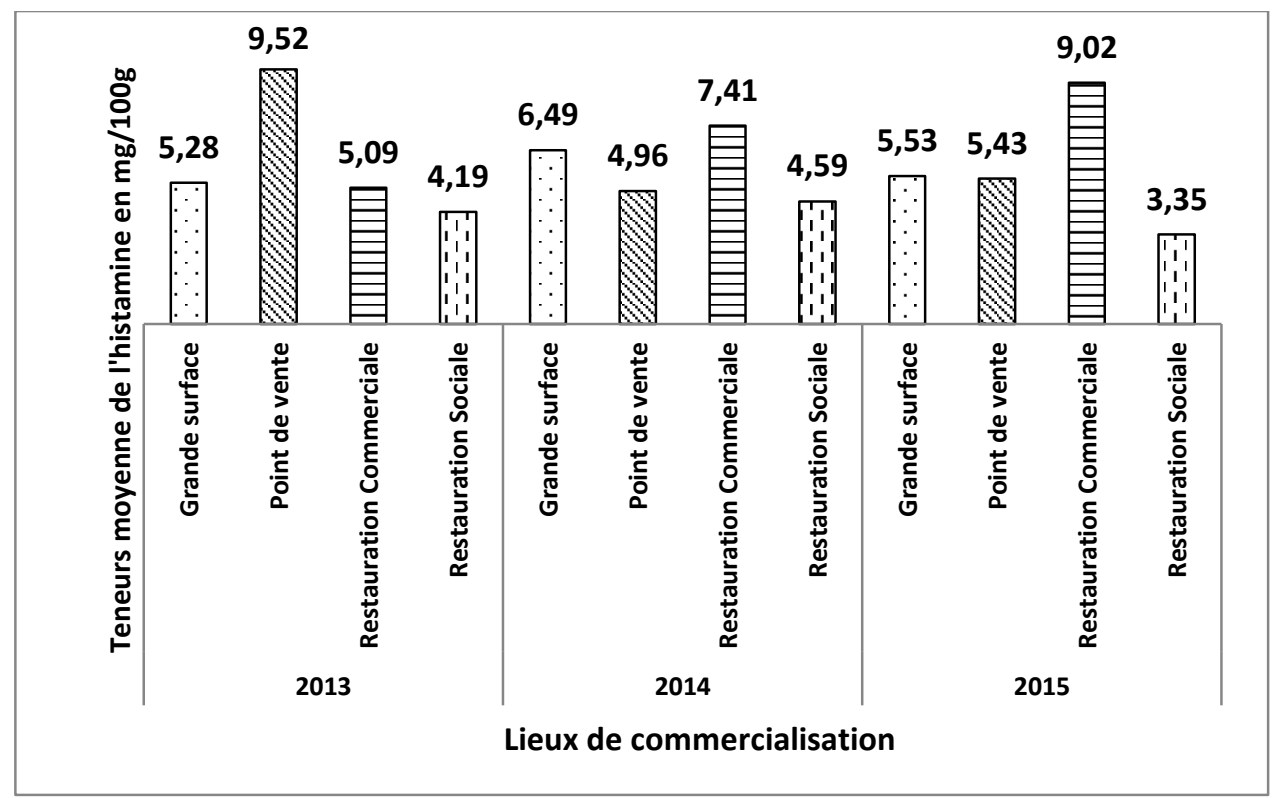

Figure 4 : Teneur moyenne en histamine en fonction des lieux de commercialisation durant 2013-2014 et 2015.

Les teneurs les plus faibles sont celles au niveau des lieux de la restauration sociale durant toutes les années. Les teneurs au niveau des grandes surfaces restent stables aux alentours d'une moyenne de 6,09 $\mathrm{mg} / 100 \mathrm{~g}$ contrairement aux teneurs au niveau des points de vente instables en fonction des années. Alors que celles des lieux de la restauration commerciale augmentent d'une année à une autre, ce qui pousse à recommander de renforcer davantage le contrôle des lieux de la restauration commerciale et des points de vente par l'autorité compétente et que ces lieux doivent mettre en place des procédures plus strictes en matière d'histamine à la réception et durant le stockage jusqu'à leur commercialisation.

\section{Evaluation semi quantitative du risque histamine pour les divers lieux de commercialisation}

Le pourcentage de non-conformité le plus élevé en matière d'histamine est trouvé dans les produits de la pêche commercialisés dans les grandes surfaces avec un taux de non conformité de 1,57\%, suivi par les points de vente avec $0,9 \%$, ensuite les lieux de la restauration commerciale avec $0,45 \%$ et en dernier lieu, les produits distribués dans les lieux de restauration sociale avec $0,22 \%$ (Tableau 10 ). 
Tableau 10 : Teneurs moyennes en histamine, taux des non conformités ainsi que le pourcentage des teneurs d'histamine par classe pour les divers lieux de vente.

\begin{tabular}{|c|c|c|c|c|c|c|c|c|}
\hline \multirow{2}{*}{ Lieux de commercialisation } & \multirow{2}{*}{$\begin{array}{c}\text { Moy } \\
\mathrm{mg} / 100 \mathrm{~g}\end{array}$} & \multirow{2}{*}{$\begin{array}{c}\mathrm{NC} \\
\%\end{array}$} & \multicolumn{6}{|c|}{$\begin{array}{l}\text { Pourcentage des teneurs d'histamine par classe } \\
\qquad(\mathrm{mg} / 100 \mathrm{~g})\end{array}$} \\
\hline & & & $\stackrel{n}{i}$ & in & $\dot{n} n$ & $\stackrel{1}{2} 0$ & 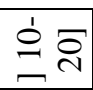 & $\stackrel{\wedge}{\wedge}$ \\
\hline Grande surface & 5,77 & 1,57 & 15,92 & 6,95 & 13,90 & 10,76 & 1,12 & 1,12 \\
\hline Point de vente & 6,09 & 0,90 & 6,50 & 3,36 & 9,42 & 5,83 & 0,22 & 0,67 \\
\hline Restauration Commerciale & 6,68 & 0,45 & 2,02 & 2,24 & 0,90 & 1,12 & 0,22 & 0,45 \\
\hline Restauration Sociale & 4,14 & 0,22 & 4,26 & 7,62 & 4,48 & 0,67 & 0,22 & 0,00 \\
\hline Total & 5,63 & 3,14 & 28,7 & 20,17 & 28,7 & 18,38 & 1,78 & 2,24 \\
\hline
\end{tabular}

Pour chaque lieu de commercialisation, les teneurs moyennes d'histamine ont été calculées, le pourcentage des non conformités a été chiffré et le pourcentage des teneurs d'histamine a été ordonnancé en six classes : a $<2,5 \mathrm{mg} / 100 \mathrm{~g}, 2,5<\mathrm{b}<5 \mathrm{mg} / 100 \mathrm{~g}, 5<\mathrm{c}<7,5 \mathrm{mg} / 100 \mathrm{~g}, 7,5<\mathrm{d}$ $<10 \mathrm{mg} / 100 \mathrm{~g}, 10<\mathrm{e}<20 \mathrm{mg} / 100 \mathrm{~g}$ et f $>20 \mathrm{mg} / 100 \mathrm{~g}$ (Tableau 11).

Tableau 11 : Evaluation semi quantitative du risque de l'histamine pour les divers lieux de commercialisation

\begin{tabular}{|c|c|c|c|c|c|c|c|c|c|c|}
\hline \multirow[b]{2}{*}{$\begin{array}{l}\text { Lieu de } \\
\text { vente }\end{array}$} & \multirow[b]{2}{*}{$\begin{array}{l}\text { Rang } \\
\text { moy }\end{array}$} & \multirow[b]{2}{*}{$\begin{array}{c}\text { Rang } \\
\text { NC } \\
\%\end{array}$} & \multicolumn{6}{|c|}{ Rangs dans les classes } & \multirow{2}{*}{$\begin{array}{c}\text { Importance } \\
\text { du Risque } \\
\text { de } \\
\text { l'histamine }\end{array}$} & \multirow[b]{2}{*}{$\begin{array}{l}\text { Classe du } \\
\text { risque }\end{array}$} \\
\hline & & & $\begin{array}{l}n \\
v \\
v\end{array}$ & $\begin{array}{l}n \\
n \\
n \\
i \\
n\end{array}$ & $\begin{array}{l}n \\
n \\
n \\
n\end{array}$ & $\stackrel{i}{\stackrel{n}{-}}=$ & 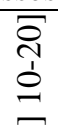 & $\underset{\wedge}{\stackrel{i}{\wedge}}$ & & \\
\hline $\begin{array}{l}\text { Grande } \\
\text { surface }\end{array}$ & 2 & 4 & 4 & 3 & 4 & 4 & 2 & 4 & $89,08 \%$ & $\begin{array}{l}\text { Risque } \\
\text { élevé }\end{array}$ \\
\hline Point de vente & 3 & 3 & 3 & 2 & 3 & 3 & 1 & 3 & $10,57 \%$ & $\begin{array}{l}\text { Risque } \\
\text { moyen }\end{array}$ \\
\hline Restauration Commerciale & 4 & 2 & 1 & 1 & 1 & 2 & 1 & 2 & $0,23 \%$ & $\begin{array}{l}\text { Risque } \\
\text { faible }\end{array}$ \\
\hline $\begin{array}{l}\text { Restauration } \\
\text { Sociale }\end{array}$ & 1 & 1 & 2 & 4 & 2 & 1 & 1 & 1 & $0,12 \%$ & $\begin{array}{c}\text { Risque } \\
\text { négligeable }\end{array}$ \\
\hline
\end{tabular}

Les produits de la pêche commercialisés dans les grandes surfaces présentent plus de risque en matière de teneur moyenne en histamine, suivis par ceux commercialisés dans les points de vente qui présentent un risque moyen, ensuite ceux vendus dans les lieux de la restauration commerciale avec un risque faible et en dernier lieu se classent les lieux de la restauration sociale avec un risque négligeable comme étant les lieux présentant les produits avec les plus faibles teneurs en histamine (Tableau 11).

Le risque plus élevé associé aux produits de grandes surfaces pourrait dans le cas de la semi-conserve s'expliquer par des durées plus longues de mise à l'étal, alors que dans les secteurs de la restauration, la rotation des stocks plus rapide est en faveur d'une moindre accumulation d'histamine.

D'après ces résultats, nous recommandons aux responsables des grandes surfaces de mettre en place des cahiers de charges avec leurs fournisseurs spécifiant les exigences de qualité surtout en matière 
d'histamine. Pour les commerçants des points de vente, il faut prévoir des sessions de sensibilisation et d'information.

\section{Histamine et diverses villes du Maroc}

La synthèse des résultats d'analyse de l'histamine en fonction des villes du Maroc a fait ressortir que les conserves commercialisés à Larache, Kenitra et Tétouan présentent les teneurs les plus faibles en matière d'histamine $(1,27 ; 1,58$ et $2,24 \mathrm{mg} / 100 \mathrm{~g}$, respectivement), alors que les conserves de poisson commercialisés à Kalaat Sraghna, Fqih Ben Saleh, Mohammedia, El Jadida et Casablanca ont les teneurs les plus élevées durant les trois années $(3,4 ; 7,92 ; 7,83 ; 7,5$ et $7,41 \mathrm{mg} / 100 \mathrm{~g}$, respectivement) (Figure 5). Ceci est de nature à confirmer les témoignages des professionnels de la filière indiquant de meilleures pratiques d'entreposage-glaçagemanutention du poisson frais destiné à la transformation débarqué dans les côtes nord en comparaison au sud.

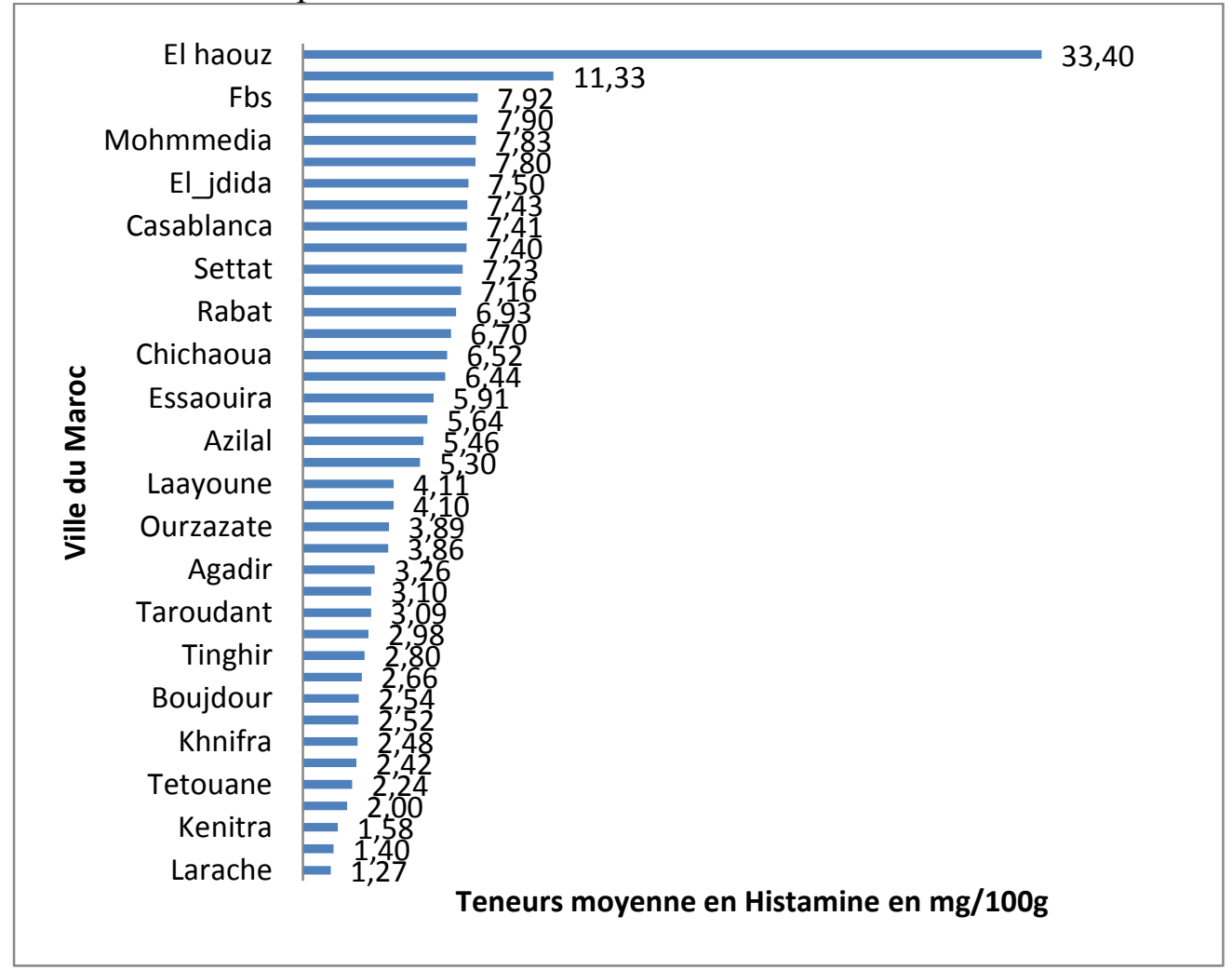

Figure 5: Histamine dans les conserves de poisson commercialisés au niveau des diverses villes au Maroc durant 2013, 2014 et 2015.

Pour les semi-conserves, les niveaux les plus faibles en histamine correspondent aux villes de M'diq-Fnideq, Larache et Tanger-Asilah $(1,28 ; 1,3$ et $1,32 \mathrm{mg} / 100 \mathrm{~g}$, respectivement $)$ en comparaison aux autres 
villes du royaume durant 2013-2014 et 2015. En revanche, les semi conserves à teneurs plus élevées sont celles commercialisées dans les villes d'Agadir, Casablanca, Mohammedia, Rabat et Marrakech (13,85; 8,88 ; 7,$07 ; 5$ et 4,20 mg/100g, respectivement). Il est à préciser que ces teneurs sont conformes eu égard à la réglementation en vigueur qui stipule que la limite de sécurité est de $20 \mathrm{mg} / 100 \mathrm{~g}$ (Figure 6). La même conclusion concernant la meilleure qualité de la matière première dans le nord s'applique aussi au contexte de la semi-conserve

Ce qui va dans le même sens des conclusions déduites en haut qui stipulent que les zones côtières nord présentent les plus faibles risques, que les semi-conserves des villes côtières ont des teneurs plus élevées par rapport à celles des zones continentales et que c'est le contraire pour les conserves.

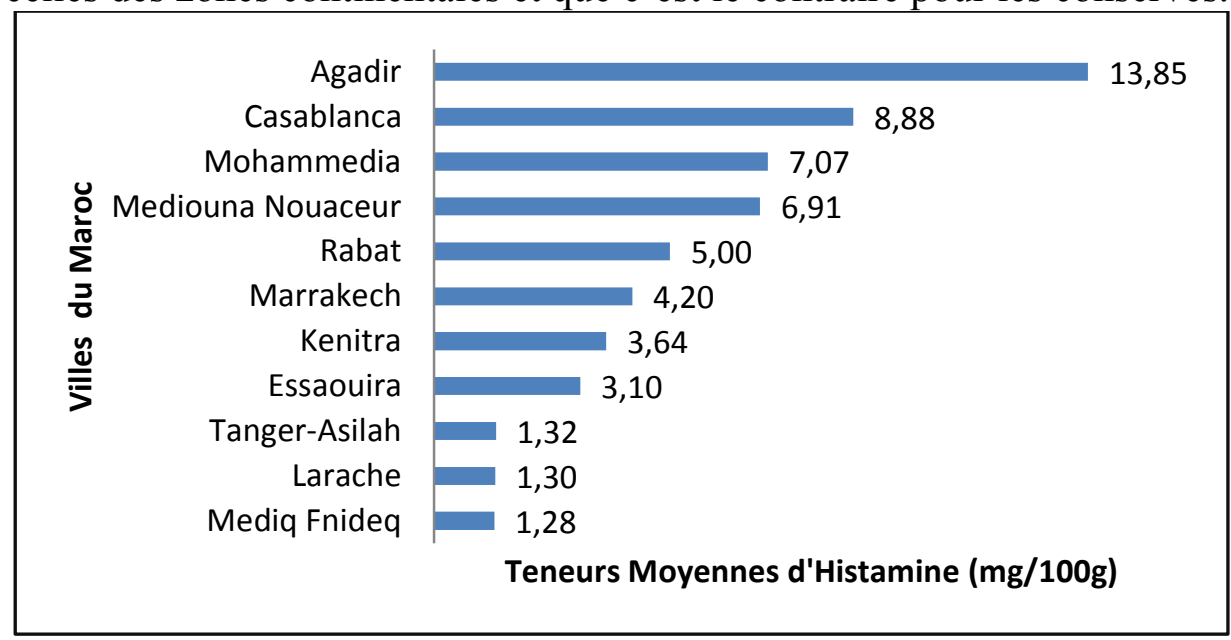

Figure 6: Histamine dans les semi conserves de poisson commercialisés au niveau des diverses villes au Maroc durant 2013, 2014 et 2015

\section{Comparaison avec d'autres études de surveillance des teneurs en histamine dans des marchés de divers pays}

Il s'agit d'une problématique de sécurité sanitaire dans plusieurs pays où plusieurs études ont été effectuées soit dans le cadre d'un échantillonnage aléatoire soit suite à des cas d'intoxications histaminiques.

Une étude menée par Ababouch (1989) sur l'occurrence de 1'histamine dans les boites de conserves de sardine a montré que la majorité des boites $(74 \%)$ contenaient de faibles teneurs en histamine $(<10 \mathrm{mg} / 100 \mathrm{~g})$, tandis que $21 \%$ contenaient entre 10 et $50 \mathrm{mg} / 100$ get que $5 \%$ contenaient des doses élevées (>50 mg/100g) susceptibles d'induire des intoxications scombroïdiques. Le taux de non-conformité était de l'ordre de $27 \%$.

Sur la base des résultats obtenus dans la présente étude, qui a fait ressortir que 95,96\% ont des teneurs en histamine inferieures à $10 \mathrm{mg} / 100 \mathrm{~g}$, et que le taux de non conformités est de l'ordre de $3,14 \%$, on pourrait 
avancer qu'il y a eu une amélioration de la situation depuis l'étude de Ababouch (1989). En effet, le Maroc a mis en place des stratégies permettant d'assurer un niveau de sécurité sanitaire élevé englobant toute la chaîne alimentaire depuis la capture jusqu'au consommateur final. Celles-ci sont basées sur une évaluation permanente des risques que représentent les matières premières et les méthodes d'exploitation, on cite à titre d'exemple, la mise à niveau du secteur en matière d'infrastructure et d'équipement, l'augmentation de la conscience et de la qualification des professionnels, la formation continue des inspecteurs, le renforcement des contrôles pour le respect de la chaine du froid et des bonnes pratiques d'hygiène et de fabrication. Le Maroc, a aussi actualisé son arsenal juridique, accompagné par la création d'une nouvelle structure (l'ONSSA) responsable exclusivement du contrôle le long de la chaine alimentaire et dont l'organisation répond aux recommandations et normes des organisations internationales auxquelles le Maroc participe activement, notamment les accords de l'OMC (SPS et OTC), le programme mixte FAO/OMS des normes alimentaires (Codex Alimentarius), l'Organisation Mondiale de la Santé Animale (OIE), etc.

Une étude effectuée sur l'incidence de l'histamine et les bactéries produisant l'histamine dans les poissons commercialisés au marché de Barcelone a révélé un taux de détection de $83,3 \%$ des échantillons de thon

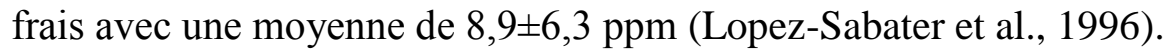

De même, une autre étude effectuée en République Tchèque sur l'occurrence de l'histamine dans du poisson frais dans des restaurants a montré que $86,6 \%$ des échantillons avaient des valeurs d'histamine inférieures à $10 \mathrm{mg} / \mathrm{kg}, 8 \%$ moins de $100 \mathrm{mg} / \mathrm{kg}$ et $5,35 \%$ supérieur à 100 $\mathrm{mg} / \mathrm{kg}$ et même ont atteint $1000 \mathrm{mg} / \mathrm{kg}$ (Bunk et al., 2013).

(Huang et al., 2010) ont conduit une étude sur le niveau des bactéries productrices d'histamine dans des produits de la pêche séchés vendus dans le marché Taïwanais et ont démontré que $69,6 \%$ des produits analysés avaient des teneurs en histamine inférieures à $50 \mathrm{mg} / \mathrm{kg}, 21,7 \%$ entre 50 et 200 $\mathrm{mg} / \mathrm{kg}$ et $8,7 \%$ entre 200 et $500 \mathrm{mg} / \mathrm{kg}$. Une autre enquête établie sur 61 échantillons de filets de maquereau vendus sur un marché de détail à Taiwan, a montré que $6,5 \%$ des échantillons ont des teneurs en histamine de 128 $288 \mathrm{mg} / \mathrm{kg}$ (Tsai et al., 2005).

En Italie, une surveillance du niveau de l'histamine effectuée de 2009 à 2011 a montré des concentrations supérieures à $2,5 \mathrm{mg} / \mathrm{kg}$ trouvés dans $58 \%$ des 311 échantillons analysés avec un taux de non-conformité de 5\%. Le plus grand nombre d'échantillons non conformes concerne les 110 échantillons d'anchois frais analysés. Les valeurs de l'histamine varient entre 2,5 et $327 \mathrm{mg} / \mathrm{kg}$ avec une moyenne de $29 \mathrm{mg} / \mathrm{kg}$ (Muscarella, 2013). 
Park et al., (2010) ont surveillé les teneurs en amines biogènes dans les poissons consommés en Corée et ont signalé une concentration moyenne d'histamine de $41,1 \mathrm{mg} / \mathrm{kg}$. Pour les anchois transformés, $75 \%$ des échantillons ont des teneurs inférieures à $50 \mathrm{mg} / \mathrm{kg}$ et le taux non-conformité était de $14 \%$.

(Zarei et al., 2011) a montré sur 40 échantillons de conserves de thon commercialisées en Iran, que les valeurs d'histamine varient entre 0,12 à $648,20 \mathrm{mg} / \mathrm{kg}$ et où $25 \%$ des échantillons contenaient plus de $50 \mathrm{mg} / \mathrm{kg}$ d'histamine, la limite permise suggérée par la FDA américaine.

Une étude menée en Inde par Joshi (Joshi, 2011), a révélé que les teneurs moyennes en histamine sont de l'ordre de $200 \mathrm{mg} / \mathrm{kg}$ sur des échantillons de maquereau, ce qui indique un risque potentiel d'intoxication à l'histamine. L'étude suggère d'améliorer la pratique des conditions d'hygiène pendant la manipulation et la transformation.

Une étude de surveillance depuis 1988 à 1991, des teneurs d'histamine dans 191 échantillons de poissons frais (sardine, thon, bonite, anchois et chinchard) commercialisés dans un marché de détail au Brésil, a démontré des teneurs oscillant entre 1,2 et $36 \mathrm{mg} / \mathrm{kg}$ (Soares et al., 1994). D'autres résultats ont été rapportés par (Silva et al., 2011) où un niveau d'histamine inférieur à $20 \mathrm{mg} / \mathrm{kg}$ a été trouvé dans des conserves de thons.

Une étude menée en Russie, a démontré que 4,7\% des 149 échantillons de poisson frais et en conserve, contenaient des teneurs en histamine dépassant la concentration maximale admissible $50 \mathrm{mg} / \mathrm{kg}$. (Lebedzinska et al., 1990).

En Pologne, la teneur en histamine dans le poisson frais et les produits de la pêche variait de zéro à 80 et de zéro à $160 \mathrm{mg} / \mathrm{kg}$, respectivement (Gajewska et al., 1991).

En Turquie, 7,7\% des 39 échantillons de conserve de poisson commercialisés dans le marché, contenait plus de $1000 \mathrm{mg} / \mathrm{kg}$ (Erkan et al., 2001). En Egypte, dans 80 échantillons de sardines, le taux de nonconformité était de $5 \%$ pour celles fraiches importées et $10 \%$ pour celles salées et en conserve produites localement (Samaha et al., 1997). Au Mexique, des concentrations d'histamine allant de 5 à $70,3 \mathrm{mg} / \mathrm{kg}$ ont été signalées dans les conserves de sardine (Pacheco et al., 2000).En NouvelleZélande, 107 échantillons de poissons fumés ont été collectés sur des marchés de détail, les résultats ont démontré que $1,9 \%$ ont dépassé le niveau de danger $(346,4$ et $681,8 \mathrm{mg} / \mathrm{kg}$ ) et que $7,5 \%$ avaient des niveaux d'histamine dépassant le niveau USFDA (Fletcher et al., 1998).

La teneur en histamine variait de 2,3 à $3,8 \mathrm{mg} / \mathrm{kg}$ dans des thons frais et de zéro à $8001 \mathrm{mg} / \mathrm{kg}$ sur des thons traitées dans 80 échantillons commercialisés au niveau des points de vente à Cape Town, en Afrique du Sud (Auerswald et al., 2006). 
Des teneurs en histamine ont été détectés suite à des intoxications histaminiques dans plusieurs pays comme le Japon (Osaka), due à la consommation de sardines séchées. La teneur en histamine mesurée était de l'ordre $1700 \mathrm{mg} / \mathrm{kg}$ (Kanki et al., 2004). Plusieurs d'autres cas d'intoxication ont été rapportés dans divers pays suite à la consommation de thon, en Taiwan, les teneurs été de $1608 \mathrm{mg} / \mathrm{kg}$ (Hwi-Chang et al., 2008), au Japon avec des teneurs supérieures à $3100 \mathrm{mg} / \mathrm{kg}$ (Ohnuma et al., 2001). Le Hareng et l'anchois ont été associés à des intoxications histaminiques (Taylor, 1986). (Bartholomew et al., 1987) a étudié 258 incidents d'intoxication histaminiques et a rapporté que parmi les poissons non scombroidiques, les poissons les plus souvent impliqués étaient le saumon (12 incidents) et le hareng (10 incidents).

Selon le site Web du Système d'alerte rapide pour les aliments et les aliments pour animaux, 66 cas d'intoxication à l'histamine dans l'Union européenne ont été enregistrés du 07/05/2008 à 07/01/2017(RASSF, 2017).

Le 26 novembre 2010, plusieurs cas d'intoxication histaminique ont eu lieu chez des militaires français en Sénégal et la concentration en histamine dans l'échantillon du thon a été $4900 \mathrm{mg} / \mathrm{kg}$, soit 50 fois la limite autorisée par la réglementation européenne (Demoncheaux et al., 2011).(Boutin et al., 1998) rapporte une épidémie d'une intoxication histaminique survenue dans la Marine due à la consommation d'espadon fumé (Xyphias gladius) d'importation. Vingt malades ont été recensés et les dosages d'histamine ont montré des teneurs allant de2030 à $4750 \mathrm{mg} / \mathrm{kg}$ dans 9 échantillons de poisson testés.

Tous ces auteurs confirment que la mauvaise qualité de la matière première et/ou des méthodes de manipulation et de traitement défectueuses sont les principales raisons des taux élevés d'histamine.

\section{Conclusion}

La teneur moyenne en histamine dans les conserves et semi conserve de poisson commercialisés au Maroc est 5,63 $\mathrm{mg} / 100 \mathrm{~g}$, elle est de 5,14 $\mathrm{mg} / 100 \mathrm{~g}$ pour les conserves de sardine $(\mathrm{TNC}=2,02 \%$ ) et de 7,76 mg/100 $\mathrm{g}$ pour les semi conserves d'anchois $(\mathrm{TNC}=1,12 \%) .95,96 \%$ des produits commercialisés sont conformes et ont des teneurs inferieures à la limite réglementaire $(100 \mathrm{mg} / 100 \mathrm{~g})(\mathrm{TNC}=3,14 \%)$. On peut conclure donc, que le consommateur marocain est pour le moment protégé.

La zone côtière centre constitue la zone présentant le risque le plus élevé en matière de teneur en histamine $(\mathrm{m}=7,11 \mathrm{mg} / 100 \mathrm{~g})$. Il en est de même pour les grandes surfaces qui constituent les lieux de commercialisation avec le plus grand risque en matière d'histamine $(\mathrm{m}=$ $5,77 \mathrm{mg} / 100 \mathrm{~g}$ ). Par contre, les lieux de la restauration sociale sont les lieux 
au plus faible risque $(\mathrm{m}=4,14 \mathrm{mg} / 100 \mathrm{~g})$ de même la zone côtière Nord est la zone ayant le risque le plus faible $(\mathrm{m}=2,11 \mathrm{mg} / 100 \mathrm{~g})$.

On peut donc avancer que le travail important réalisé par l'autorité compétente marocaine (Service Vétérinaires sur le terrain en collaboration avec l'Administration Centrale de l'ONSSA et ses Directions Régionales), associé à l'engagement des professionnels pour la sécurité et la qualité de leurs produits, participent efficacement à consolider la confiance du consommateur, à maintenir un excellent niveau de sécurité sanitaire des aliments commercialisés sur le marché marocain et contribue en même temps à la valorisation des produits de la pêche marocains et l'amélioration de leurs compétitivité au niveaux des marchés internationaux.

\section{References :}

1. Ababouch, L., \& Afilal, M. E. (1989). L'histamine dans la sardine marocaine fraîche et en conserves. Evolution au cours du stockage sous glace et en présence de sel. Mircen Journal, , 77-86.

2. Anguilar, P., Sanchez, L., \& Ozuna, V. (2000). Histamine quantification in Monterey sardine muscle and canned products from north-western Mexico. Journal of Food Composition And Analysis , 188-195.

3. Auerswald, L.,Morren, C.,\& Lopata. (2006).Histamine levels in seventeen species of fresh and processed South African seafood. Food Chemical,231-239.

4. Bartholomew, B., Berry, P., Rodhouse, J., \& Gilbert, R. (1987). Scombrotoxic fish poisoning in Britain: features of over 250 suspected incidents from 1976 to 1986. Epidemiology and Infection , 775-782.

5. Boutin, J., Puyhardy, J., Chianea, D., Andreu, P., Paez, S., Fize, L., et al. (1998). Les intoxications alimentaires histaminiques. Santé publique , 29-37.

6. Bunka, F., Budinsky, P., Zimakov, B., Mehaut, M., \& Flasarova, R. (2013). Biogenic amines occurrence in fish meat sampled from restaurants in region. Food Control , 49-52.

7. CE. (2005, Novembre 15). Réglement (CE) $\mathrm{N}^{\circ} 2073 / 2005$ de la Commission du 15 novembre 2005 concernant les critères microbiologiques applicables aux denrées alimentaires.

8. Demoncheaux, J.-P., Mazenot, C., \& Rémy, M. (2011). Intoxication massive à l'histamine après consommation de thon jaune. BEH , 4546.

9. Emilio I. Lopez-Sabater, J. J.-J.-H.-V. (1996). Incidence of histamine-forming bacteria and histamine content in scombroid fish 
species from retail markets in the Barcelona area. International Journal of Food Microbiology , 411-418.

10. Erkan, N., Helle, N., \& Ozden, O. (2001). the content of biogenic amines in canned fish from turkish market. Berl Munch Tierarztl Wochenschr, 241-245.

11. FAO/WHO. (2013). Expert Meeting on the Public Health Risks of Histamine and other Biogenic Amines from Fish and Fishery Products. Rome Italy: Food and Agriculture Organization of the United Nations.

12. FDA. (2011). Fish and Fishery Products Hazards and Controls Guidance Fourth Edition. Department of Health and Human Services Public Health Service -Food and Drug Administration -Center for food safety and applied nutrition Office of Food Safety.

13. Fletcher, G., Summers, G., \& Veqhe, V. (1998). Levels of histamine and histamine-producing bacteria in smoked fish from New Zealand. Food protection , 1064-1070.

14. Frantisek Bunka, P. B. (2013). Biogenic amines occurrence in fish meat sampled from restaurants in region. Food Control , 49-52.

15. Huang, Y.-R., Liu, K.-J., Hseih, H.-S., Hsieh, C.-H., \& Hwang, D.-F. (2010). Histamine level and histamine-forming bacteria in dried fish products sold. Food Control , 1234-1239.

16. Hwi-Chang, C., Hsien-Feng, K., Wen-Chieh, C., Wen-feng, L., Deng-Fwu, H., Yi-Chen, L., et al. (2008). Determination of histamine and histamine-forming bacteria in tuna dumpling implicated in a food-borne poisoning. Food Chemistry , 612-618.

17. Joshi, P., \& Vishal, B. (2011). Study of Histamine Forming Bacteria in Commercial fish samples of Kalyan city. Current SciDirect Publication , $39-42$.

18. Kanki, M., Yoda, T., Ishibashi, M., \& Tsukamoto, T. (2004). Photobacterium phosporeum caused a histamine fish poisoning outbreak. Journal Of Food Microbiology , 79-87.

19. Lebedzinka, A., Eller, K., \& Tutellian, V. (1990). Biogenic amine contents in fish and fish preserves. Vopr Pitan , 47-51.

20. Lopez-Sabater, E., Rodriguez-Jerez, J., Hernandez-Herrero, M., \& Mora-Ventura, M. (1996). Incidence of histamine-forming bacteria and histamine content in scombroid fish species from retail markets in the Barcelona area. International Journal of Food Microbiology, 411-418.

21. Manzano, M., Anguilar, P., Rojas, D., \& Sanchez, L. (2000). Post mortem changes in black skipjack muscle during storage in ice. Journal of Food Science, 65: . , 774-779. 
22. Marilena Muscarella, S. L. (2013). Survey of histamine levels in fresh fish and fish products collected in Puglia (Italy). Food Control, 211-217.

23. Muscarella, M., Lo Magro, S., Campaniello, M., \& Armentano, A. (2013). Survey of histamine levels in fresh fish and fish products collected in Puglia (Italy). Food Control , 211-217.

24. Ohnuma, S., Higa, M., Hamanaka, S., Matsushima, K., \& Yamamuro, W. (2001). An outbreak of allergy-like food poisoning. InternalMedicine , 833-835.

25. Olley, J., \& Lehane, L. (2000). Histamine fish poisoning revisited. International Journal of Food Microbiology , 1-37.

26. ONSSA. (2016, Décembre 31). Les attributions et les missions. Consulté le Décembre 31, 2016, sur http://www.onssa.gov.ma/fr/onssa-sp-5790/missions

27. Ouadghiri, A. E. (2016, Juillet 15). Agripeche l'Actualité Economique du Maroc. Consulté le Decembre 31, 2016, sur FAO: Le Maroc premier producteur/exportateur mondial de la sardine: http://agripeche.com/3225-fao-le-maroc-premierproducteurexportateur-mondial-de-la-sardine.html

28. Pacheco-Aguilar R, L.-S. M.-O. (2000). Histamine quantification in Monterey sardine muscle and canned products rom north-western Mexico. Journal of Food Composition And Analysis , 188-195.

29. Park, J. s., Lee, C. H., Kwon, E. Y., Lee, H. J., \& Kim, J. Y. (2010). Monitoring the contents of biogenic amines in fish and fish products consumed in Korea. Food Control , 1219-1226.

30. RASSF. (2017, Janvier 8). Commission Européenne. Consulté le Janvier 8, 2017, sur RASFF Portal: https://webgate.ec.europa.eu/rasffwindow/portal/?event=searchResultList

31. Samaha, I., Elgazzar, M., \& El-Atabay. (1997). Histamine content in sardine and its products. Egypt Public Hlth , 471-478.

32. Silva, T., Sabaini, P., Warlley, E., \& Gloria, M. B. (2011). Occurrence of histamine in Brazilian fresh and canned tuna. Food Control , 323-327.

33. Soares, M., Gloria, A., Valeria, F., \& Beatriz, M. (1994). Histamine levels in canned fish available in Belo Horizonte, Minas Gerais, Brasil. Journal Food Of Composition , 102-109.

34. Tarliane M. Silva, P. S. (2011). Occurrence of histamine in Brazilian fresh and canned tuna. Food Control , 323-327.

35. Taylor, S. L. (1986). Histamine food poisonning: Toxicology and clinical aspects. Crit Rev Toxicol , 91-128. 
36. Taylor, S. L., \& Stratton, J. E. (1989). Hstamine poisoning (Scombroid fish poisoning): An allergy-like intoxication. Clinical Toxicology , 225-240.

37. Trade, F. (2016, decembre 31). Fellah Trade. Consulté le Décembre 1, 2016, sur http://www.fellah-trade.com/fr/filiere-animale/chiffrescles-peche: http://www.fellah-trade.com/fr/filiere-animale/chiffrescles-peche

38. Tsai, Y.-H., Lin, C.-Y., Chang, S.-C., \& Chen, H.-C. (2005). Occurrence of histamine and histamine-forming bacteria in salted mackerel in Taiwan. Food Microbiology , 461-467.

39. Yung-Hsiang TsaI, C.-Y. L.-C.-C. (2005). Occurrence of histamine and histamine-forming bacteria in salted. FOOD MICROBIOLOGY, 461-467.

40. Yu-Ru Huang, K.-J. L.-S.-H.-F. (2010). Histamine level and histamine-forming bacteria in dried fish products sold. Food Control , 1234-1239.

41. Zarei, M., Najafzadeh, H., Enayati, A., \& Pashmforoush, M. (2011). Biogenic Amines Content of Canned Tuna Fish Marketed in Iran. American-Eurasian Journal of Toxicological Sciences , 190-193. 